\title{
VARIETIES OF NILPOTENT GROUPS OF CLASS FOUR (II)
}

\author{
PATRICK FITZPATRICK
}

(Received 3 April 1981)

Communicated by D. E. Taylor

\begin{abstract}
The first paper (written jointly with L. G. Kovács) of this three-part series reduced the problem of determining all varieties of the title to the study of the varieties of nilpotent groups of class (at most) four whose free groups have no nontrivial elements of odd order. The present paper deals with these under the additional assumption that the variety contains all nilpotent groups of class three. We label each such variety by a vector of eleven parameters, each parameter a nonnegative integer or $\infty$, subject to numerous but simple conditions. Each vector satisfying these conditions is in fact used, and matches directly a (finite) defining set of laws for the variety it labels. Moreover, one can readily recognize from the parameters whether one variety is contained in another. The third paper will complete the determination of all varieties of nilpotent groups of class four.
\end{abstract}

1980 Mathematics subject classification (Amer. Math. Soc.): 20 E 10.

\section{Introduction}

The determination of varieties of nilpotent groups of class four was considered in Fitzpatrick and Kovács (1982) where it was shown that the problem splits into two parts: the case of 2-torsionfree varieties (that is, varieties whose free groups have no element of order 2) and the case of 2 -torsionfree varieties (whose free groups have no nontrivial elements of odd order). The first case was dealt with completely in that paper; we begin an analysis of the second by finding those 2'-torsionfree subvarieties of $\mathfrak{R}_{4}$ containing $\mathfrak{R}_{3}$. (All varietal notation follows Hanna Neumann (1967) unless otherwise stated.)

As usual all the work will be carried out in the dual context; we wish to determine those 2 -isolated fully invariant subgroups of the rank 4 free group $F$ of $\mathfrak{R}_{4}$ which are contained in the last nontrivial term $N_{3}=\mathfrak{R}_{3}(F)$ of its lower

C. Copyright Australian Mathematical Society 1983 
central series. (A normal subgroup is $2^{\prime}$-isolated if its factor group has no nontrivial elements of odd order and isolated if its factor group is torsionfree.)

Let $\{x, y, z, t\}$ be a free generating set of $F$. We choose certain endomorphisms of $F$, namely: the 24 which permute these free generators; the endomorphism which maps $x$ to $x y$ and leaves each of $y, z, t$ fixed; and, for each integer $\kappa$, the endomorphism which maps $x$ to $x^{\star}$ and leaves $y, z, t$ fixed. The restrictions of these endomorphisms generate the semigroup of all endomorphisms of the commutator factor group $F / F^{\prime}$ (see Lemma 3.1). Observe that any two endomorphisms of $F$ which agree on $F / F^{\prime}$ also agree on $N_{3}$. This means that a subgroup of $\mathrm{N}_{3}$ is fully invariant in $\mathrm{F}$ if and only if it admits the distinguished endomorphisms listed above. In fact, as a group with the endomorphisms of $F$ as operators $N_{3}$ may as well be viewed as a module for the semigroup of all endomorphisms of $F / F^{\prime}$. With reference to the basis $\left\{x F^{\prime}, y F^{\prime}, z F^{\prime}, t F^{\prime}\right\}$ of $F / F^{\prime}$, that semigroup is just the multiplicative semigroup $G=\operatorname{Mat}^{\times}(4, Z)$ of all $4 \times 4$ matrices with integer entries. As our interest lies in the 2 '-isolated submodules of $N_{3}$ (those whose factor modules are 2 '-isolated as abelian groups), it is convenient to tensor it (over $Z$ ) with the ring $Z_{(2)}$ of rational numbers with odd denominators (the localization of $Z$ at 2 ) and look for submodules in this tensor product. Thus we end up investigating modules over the semigroup algebra $Z_{(2)} G$.

The Magnus-Witt argument elaborated in Wall (1978) and more specifically in Kovács (1978) allows us, with a few minor modifications, to restate this problem in the following more convenient formulation. Let $L$ be the free Lie algebra of rank 4 over $Z_{(2)}$, freely generated by the "variables" $x, y, z, t$. (This may be envisaged within the algebra of all polynomials in these noncommuting variables with coefficients from $Z_{(2)}$. With respect to the usual Lie product $[u, v]$ defined as $u v-v u$ this is also a Lie algebra and $L$ is its Lie subalgebra generated by $x, y, z$, t.) As we shall not need to use associative products here we shall simply use juxtaposition for Lie products and omit left normed brackets: thus we write $x y z$ for $[[x, y], z]$. We shall be particularly interested in the set $W$ of those elements of $L$ which as polynomials are homogeneous of (total) degree 4 ; this is a free

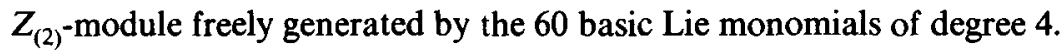

Our semigroup $G$ acts on $L$ by linear homogeneous substitutions: the matrix $\left(\alpha_{i j}\right)$ mapping $x$ to $\alpha_{11} x+\alpha_{12} y+\alpha_{13} z+\alpha_{14} t$ and so on. It is clear that $W$ is a $G$-submodule of $L$ annihilated by the zero of $G$ so $W$ is a module for the (contracted) semigroup algebra $Z_{(2)} G$; for a definition see Section 3 . The Magnus-Witt argument mentioned above now yields that the lattice $\delta(W)$ of submodules of $W$ is isomorphic to the lattice of those 2 '-isolated fully invariant subgroups of $\mathrm{F}$ contained in $\mathrm{N}_{3}$.

Our aim then is to find the $Z_{(2)} G$-submodules of $W$ and make the appropriate translation back to $F$. In fact we obtain a great deal more information than is 
really necessary for this specific task. Namely, we give a complete description of the quotient of $Z_{(2)} G$ modulo the annihilator Ann $W$ of $W$, from which we derive our results on submodules. After translation to $F$ these can be summarised as follows: $N_{3}$ contains two proper nontrivial isolated fully invariant subgroups, which of course correspond to the two torsionfree varieties (that is, varieties whose free groups are torsionfree) $\mathfrak{R}_{3}^{(2)} \cap \mathfrak{R}_{4}$ and $\mathfrak{H}^{2} \cap \mathfrak{R}_{4}$ lying between $\mathfrak{R}_{3}$ and $\mathfrak{R}_{4}$. Each 2 -isolated fully invariant subgroup contained in $N_{3}$ is labelled by a vector of 11 parameters, each parameter a non-negative integer or $\infty$, subject to simple but numerous conditions. Each vector satisfying the conditions does in fact correspond to such a subgroup and from it we can directly read off a set of fully invariant subgroup generators for the subgroup it labels. For any such subgroup $H$ we can also determine its isolator $H_{0}$ (the smallest isolated fully invariant subgroup containing it) and give an upper estimate for the exponent of the quotient $H_{0} / H$. Thus our description fulfills the requirements of the programme outlined in Section 2 of Fitzpatrick and Kovács (1982). One can also recognise from the parameters whether one subgroup is contained in another.

As far as the problem mentioned in the first paragraph of this introduction is concerned, we have left outstanding only the determination of the 2 -isolated fully invariant subgroups of $F$ not contained in $N_{3}$ : this will be taken up in a subsequent paper.

Most of the material for this paper is taken from the author's thesis [Fitzpatrick (1980)]. Financial support from the Australian National University is gratefully acknowledged. I would like also to thank my supervisor Dr L. G. Kovács under whose patient guidance this work was carried out.

\section{Associative rings and algebras}

We begin by collecting some facts concerning associative rings and algebras. Each associative ring considered will have a multiplicative identity element normally denoted by 1 which acts identically on all the modules we look at (usually right modules). For such a ring $R$ we denote by $\operatorname{Mat}(n, R)$ the ring of $n \times n$ matrices over $R$. The symbol $e_{n}(i, j)$ stands for the $n \times n$ elementary matrix with all entries 0 apart from the $(i, j)$ entry which is 1: the context will make clear to which ring 0 and 1 belong. The $n \times n$ identity matrix will also be denoted by 1 and the diagonal matrix $\operatorname{diag}\left(r_{1}, \ldots, r_{n}\right)$ is the $n \times n$ matrix with $(i, i)$ entry $r_{i}$ (for $1 \leqslant i \leqslant n$ ) and all other entries 0 .

2.1. Proposition. Let $R$ be an associative ring, $\varepsilon$ an idempotent element of $R$ such that $\operatorname{R\varepsilon } R=R$, and $M$ any right $R$-module. Then the lattice $\delta(M)$ of 
submodules of $M$ is isomorphic to the lattice $\delta(M \varepsilon)$ of submodules of the $\varepsilon R \varepsilon-$ module $M \varepsilon$.

Proof. The assumption $R \varepsilon R=R$ means that the identity 1 of $R$ may be written as a finite $\operatorname{sum} \sum a_{i} \varepsilon b_{i}$ with $a_{i}, b_{i} \in R$.

Define

$$
\begin{array}{ll}
\varphi: \delta(M) \rightarrow \delta(M \varepsilon), & X \mapsto X \varepsilon, \\
\psi: \delta(M \varepsilon) \rightarrow \delta(M), & Y \mapsto Y R .
\end{array}
$$

If $X \in \delta(M)$ and $x \in X$, we have $x=x \sum a_{i} \varepsilon b_{i}=\Sigma x a_{i} \varepsilon b_{i}$; as $x a_{i} \in X$ this shows $x \in X \varepsilon R$. Thus $X \varepsilon R \geqslant X$. The converse inclusion is obvious, so $X \varepsilon R=X$, and $\varphi \psi$ is the identity map on $\delta(M)$. If $Y \in \delta(M \varepsilon)$, then $Y=Y \varepsilon$ (because $\varepsilon^{2}=\varepsilon$ ) and so $Y=Y \varepsilon R \varepsilon=Y R \varepsilon$ : thus $\psi \varphi$ is the identity on $\delta(M \varepsilon)$. It is clear that $\varphi$ and $\psi$ preserve order, and well known that any poset-isomorphism of lattices is a lattice isomorphism.

2.2. REMARK. If $Y \in \delta(M \varepsilon)$ and $\mathscr{Y}$ is an $\varepsilon R \varepsilon$-generating set for $Y$ then $\mathscr{Y}$ is also an $R$-generating set for $Y R$.

Such an idempotent will frequently arise from a ring homomorphism

$$
\bigoplus_{k=1}^{s} \operatorname{Mat}(n(k), Z) \rightarrow R
$$

Giving a homomorphism like this amounts to specifying elements $\varepsilon_{i j}^{k}$ in $R$, with $k$ ranging from 1 to $s$ while for any fixed value of $k$ the subscripts $i, j$ range from 1 to $n(k)$, satisfying the relations

$$
\varepsilon_{i j}^{k} \varepsilon_{l m}^{n}=\delta_{k n} \delta_{j l} \varepsilon_{i m}^{k}
$$

(where $\delta_{k n}$ and $\delta_{j l}$ are Kronecker deltas). Without loss of generality we may take

$$
\sum \sum \varepsilon_{i i}^{k}=1 \text { : }
$$

for if this is not the case we may put $\varepsilon_{11}^{s+1}=1-\sum \sum \varepsilon_{i i}^{k}$ and extend the range of the superscript $k$. Then define $\varepsilon$ as

$$
\varepsilon=\sum \varepsilon_{11}^{k} .
$$

Clearly, $\varepsilon$ is an idempotent, and

$$
1=\sum \sum \varepsilon_{i i}^{k}=\sum \sum \varepsilon_{i 1}^{k} \varepsilon \varepsilon_{1 i}^{k}
$$

shows that $R \varepsilon R=R$, so 2.1 may be applied with this choice of $\varepsilon$.

Now let $K$ be a commutative (and associative) ring with 1 and let $A$ be an algebra over $K$. The next result shows how if $\varepsilon$ is an element of $A$ satisfying 2.3, 2.4 and 2.5, then a set of algebra generators of $A$ leads to a set of generators for $\varepsilon A \varepsilon$. 
2.6. Lemma. Suppose the subset $\mathcal{G}$ of $A$ generates $A$ as $K$-algebra, and 2.3, 2.4, 2.5 hold. Then the set

$$
\bigcup_{i, j, k, m} \varepsilon_{1 i}^{k} \mathcal{G}_{j 1}^{m}
$$

generates $\varepsilon A \varepsilon$ as $K$-algebra.

Proof. We need to show that if $p$ is any product of elements of $\mathcal{G}$ then $\varepsilon p \varepsilon$ is a linear combination of elements from the proposed generating set. When $p$ is just $r s$ with $r, s \in \mathcal{G}$, we have

$$
\varepsilon p \varepsilon=\varepsilon r s \varepsilon=\left(\sum \varepsilon_{11}^{l}\right) r\left(\sum \sum \sum \varepsilon_{i 1}^{k} \varepsilon_{1 i}^{m}\right) s\left(\sum \varepsilon_{11}^{n}\right)
$$

since the middle factor on the right hand side is 1 . This is a sum of products of the form $\varepsilon_{11}^{l} r \varepsilon_{i 1}^{k}$ and $\varepsilon_{1 i}^{m} s \varepsilon_{11}^{n}$, each of which lies in the proposed generating set. When $p \in \mathcal{G}$ or $p$ is a product of more than two factors from $\mathcal{G}$, the argument follows the same pattern.

In the opposite direction, if we know the algebra $\varepsilon A \varepsilon$, its elements $\varepsilon_{11}^{k}$ and the positive integers $n(k)$ then $A$ can be explicitly reconstructed as a subalgebra of $\operatorname{Mat}(n, \varepsilon A \varepsilon)$ where $n=\sum_{k=1}^{s} n(k)$. In this matrix algebra it is convenient to index rows and columns by

$$
\left\{\begin{array}{l}
k \\
i
\end{array} \mid 1 \leqslant k \leqslant s, 1 \leqslant i \leqslant n(k)\right\},
$$

write the elementary matrices as $e_{i j}^{k l}$ with multiplication rule

$$
e_{i j}^{k l} e_{i^{\prime} j^{\prime}}^{k^{\prime} l^{\prime}}=\delta_{l k^{\prime}}, \delta_{j i^{\prime}} e_{i j^{\prime}}^{k l^{\prime}}
$$

and denote a general matrix by $\left(\alpha_{i j}^{k l}\right)$ where of course

$$
\left(\alpha_{i j}^{k l}\right)=\sum \sum \sum \sum \alpha_{i j}^{k l} e_{i j}^{k l}
$$

It is straightforward to check that within $\operatorname{Mat}(n, \varepsilon A \varepsilon)$ the subset

$$
\left\{\left(\alpha_{i j}^{k l}\right) \mid \alpha_{i j}^{k l} \in \varepsilon_{11}^{k}(\varepsilon A \varepsilon) \varepsilon_{11}^{l} \text { for all } i, j, k, l\right\}
$$

is a subalgebra, $\bar{A}$ say. Our claim is then

2.7. Proposition. $\bar{A}$ is isomorphic to $A$.

Proof. The map $\varphi: A \rightarrow \bar{A}$ given by

$$
\varphi: \alpha \mapsto\left(\alpha_{i j}^{k l}\right)
$$


where $\alpha_{i j}^{k l}=\varepsilon_{1 i}^{k} \alpha \varepsilon_{j 1}^{l}$ (which belongs to $\varepsilon_{11}^{k} A \varepsilon_{11}^{l}$ because it is $\varepsilon_{11}^{k} \varepsilon_{1 i}^{k} \alpha \varepsilon_{j 1}^{l} \varepsilon_{11}^{l}$ ) is an isomorphism with two sided inverse $\psi: \bar{A} \rightarrow A$ given by

$$
\psi:\left(\alpha_{i j}^{k l}\right) \mapsto \sum \sum \sum \sum \varepsilon_{i 1}^{k} \alpha_{i j}^{k l} \varepsilon_{1 j}^{l}
$$

The details are left as an exercise.

It is in this context that we shall describe the algebra $Z_{(2)} G /$ Ann $W$ mentioned in the introduction.

\section{Preliminary reduction}

We now focus our attention on $K G=K \operatorname{Mat}^{\times}(4, Z)$. This (contracted) semigroup algebra is defined as the $K$-module freely generated by the nonzero elements of $G$, with multiplication defined by $K$-linear extension of the multiplication in $G$, after identification of the zero of $G$ with the zero of the module $K G$. Explicitly, the elements of $K G$ are the formal expressions $\sum \kappa_{g} g$ with summation over all nonzero $g$ in $G$, the $\kappa_{g}$ elements of $K$, all but finitely many of them zero. They are manipulated according to the rules

$$
\kappa \sum \alpha_{g} g+\sum \beta_{g} g=\sum\left(\kappa \alpha_{g}+\beta_{g}\right) g
$$

and

$$
\left(\sum \alpha_{g} g\right)\left(\sum \beta_{g} g\right)=\sum \gamma_{g} g
$$

where $\gamma_{g}=\Sigma \alpha_{g^{\prime}} \beta_{g^{\prime \prime}}$, summation being over all ordered pairs $\left(g^{\prime}, g^{\prime \prime}\right)$ with $g^{\prime} g^{\prime \prime}=$ $g$. We identify the nonzero elements of $G$ and the formal expressions with one coefficient 1 and all other coefficients 0 . Thus the identity of $G$ is also the identity of $K G$.

First we state an elementary result which was used implicitly in the introduction.

3.1. LeMMA. The semigroup $G$ is generated by the set $\mathcal{G}$ which consists of the $4 \times 4$ permutation matrices, the diagonal matrices $\mu_{\kappa}$ defined by $\mu_{\kappa}=\operatorname{diag}(\kappa, 1,1,1)($ one for each $\kappa$ in $Z$ ), and the matrix $\tau$ obtained from the $4 \times 4$ identity matrix by changing its $(1,2)$ entry to 1 .

Proof. This is a straightforward consequence of the familiar fact that every integer matrix can be transformed to diagonal form by elementary row and column operations, that is, by pre- and post-multiplication by products formed from $\tau, \mu_{-1}$ and the permutation matrices. All diagonal matrices are obviously in 
the semigroup generated by the $\mu_{\kappa}$ and the permutation matrices so our claim follows.

It will lead to no confusion if we write 1 for the identity of $G$. We shall also write

$$
\begin{gathered}
\sigma_{2}=\left(\begin{array}{llll}
0 & 1 & 0 & 0 \\
1 & 0 & 0 & 0 \\
0 & 0 & 1 & 0 \\
0 & 0 & 0 & 1
\end{array}\right), \quad \sigma_{3}=\left(\begin{array}{llll}
0 & 1 & 0 & 0 \\
0 & 0 & 1 & 0 \\
1 & 0 & 0 & 0 \\
0 & 0 & 0 & 1
\end{array}\right), \\
\sigma_{4}=\left(\begin{array}{llll}
0 & 1 & 0 & 0 \\
0 & 0 & 1 & 0 \\
0 & 0 & 0 & 1 \\
1 & 0 & 0 & 0
\end{array}\right),
\end{gathered}
$$

$S_{1}$ for the group consisting of 1 alone,

$S_{2}$ for the group generated by $\sigma_{2}$,

$S_{3}$ for the symmetric group generated by $\sigma_{2}$ and $\sigma_{3}$, and

$S_{4}$ for the symmetric group generated by $\sigma_{2}$ and $\sigma_{4}$.

We put $\partial_{1}=\mu_{0}$ and $\partial_{i}=\sigma_{i} \mu_{0} \sigma_{i}^{-1}$ for $i=2,3,4$; thus for instance $\partial_{3}=$ $\operatorname{diag}(1,1,0,1)$.

Our next task is to identify elements of $K G$ which satisfy the relations 2.3. Of course, the $e_{4}(i, j)$ of $G$ would always do, but as these annihilate $W$, they are no help. So we must look for others; to this end we exploit first the pairwise commuting idempotents $\partial_{1}, \ldots, \partial_{4}$. Formal expansion in $K G$ gives

$$
1=\prod_{i=1}^{4}\left[\left(1-\partial_{i}\right)+\partial_{i}\right]=\sum_{J} \prod_{j \in J}\left(1-\partial_{j}\right) \prod_{i \notin J} \partial_{i}
$$

with summation over all subsets $J$ of $\{1,2,3,4\}$. Put

$$
\varepsilon_{J}=\prod_{j \in J}\left(1-\partial_{j}\right) \prod_{i \notin J} \partial_{i}
$$

the fact that the $\partial_{i}$ are pairwise commuting idempotents immediately yields that the $\varepsilon_{J}$ are pairwise orthogonal idempotents. As $\varepsilon_{\varnothing}=0$ we restrict attention to nonempty $J$. It will be convenient to name the subsets $\{1, \ldots, i\}$ as $Z_{i}$, and to write $\varepsilon_{i}$ for $\varepsilon_{Z_{i}}$.

For subsets $I, J$ of equal cardinality, let $\sigma(I, J)$ denote that permutation of $Z_{4}$ which maps $I$ to $J$ preserving order, and $Z_{4} \backslash I$ to $Z_{4} \backslash J$ also preserving order; we shall write $\sigma(I, J)$ also for the corresponding permutation matrix. Note that $\sigma(I, J)=\sigma(J, I)^{-1}$. Next, define $\varepsilon(I, J)$ by $\varepsilon(I, J)=\varepsilon_{I} \sigma(I, J)$. We shall show that these elements satisfy 2.3 , but first we prove the following.

3.2. Lemma. Let $\sigma \in S_{4}$. Then $\varepsilon_{J} \sigma=\sigma \varepsilon_{J \sigma}$. 
Proof. It is clear that $\partial_{i} \sigma=\sigma \partial_{i \sigma}($ note the dual use of the symbol $\sigma)$ : thus

$$
\begin{aligned}
{\left[\prod_{j \in J}\left(1-\partial_{j}\right) \prod_{i \notin J} \partial_{i}\right] \sigma } & =\sigma\left[\prod_{j \in J}\left(1-\partial_{j \sigma}\right) \prod_{i \notin J} \partial_{i \sigma}\right] \\
& =\sigma\left[\prod_{j \in J \sigma}\left(1-\partial_{j}\right) \prod_{i \notin J \sigma} \partial_{i}\right],
\end{aligned}
$$

and this is what we want.

We shall make frequent use of this lemma and its obvious corollaries $\sigma \varepsilon_{J}=\varepsilon_{J_{0}-1} \sigma$ and $\varepsilon(I, J)=\sigma(I, J) \varepsilon_{J}$, without specific reference.

3.3. Proposition. For all subsets $I, J, M, N$ of $Z_{4}$,

$$
\varepsilon(I, J) \varepsilon(M, N)=\delta_{J M} \varepsilon(I, N)
$$

$\left(\delta_{J M}\right.$ a Kronecker delta $)$.

Proof. $\varepsilon(I, J) \varepsilon(M, N)=\varepsilon_{I} \sigma(I, J) \varepsilon_{M} \sigma(M, N)=\sigma(I, J) \varepsilon_{J} \varepsilon_{M} \sigma(M, N)$ and this is zero unless $J=M$. If this is the case then the cardinalities $|I|,|J|,|M|$, $|N|$ are all equal and $\varepsilon(I, J) \varepsilon(J, N)=\varepsilon_{I} \sigma(I, J) \varepsilon_{J} \sigma(J, N)=\sigma(I, J) \varepsilon_{J} \varepsilon_{J} \sigma(J, N)$ $=\sigma(I, J) \varepsilon_{J} \sigma(J, N)=\varepsilon_{I} \sigma(I, J) \sigma(J, N)=\varepsilon_{I} \sigma(I, N)=\varepsilon(I, N)$ since $\sigma(I, J) \sigma(J, N)=\sigma(I, N)$. This completes the proof.

For an application of 2.3 and so on, we may view the $\varepsilon(I, J)$ as having subscripts $I, J$ and the common cardinality of $I$ and $J$ as superscript. Since $J=M$ implies $|J|=|M|$ we may omit the superscript and the other Kronecker delta $\boldsymbol{\delta}_{|J||M|}$ required in 2.3.

Observe that $\varepsilon(I, I)=\varepsilon_{I} \sigma(I, I)=\varepsilon_{I}$ since $\sigma(I, I)$ is the identity permutation, so $\Sigma_{I} \varepsilon(I, I)=\Sigma_{I} \varepsilon_{I}=1$, corresponding to 2.4 .

The obvious choices for the subsets corresponding to the subscript 1 in 2.5 are the initial subsets $Z_{i}, 1 \leqslant i \leqslant 4$. Then for

$$
\varepsilon=\sum_{i=1}^{4} \varepsilon_{i}
$$

we have $(K G) \varepsilon(K G)=K G$ and thus we may apply 2.1 .

The only remaining task is to describe explicitly the generating set of $\varepsilon K G \varepsilon$ obtained from $\mathcal{G}$ by 2.6 .

3.4. Proposition. The union of the sets $\varepsilon_{i} S_{i} \varepsilon_{i}(1 \leqslant i \leqslant 4)$,

$$
\left\{\varepsilon_{i} \mu_{\kappa} \varepsilon_{i}, \varepsilon_{i} \tau \varepsilon_{i} \mid 1 \leqslant i \leqslant 4, \kappa \in Z\right\}
$$


and

$$
\left\{\varepsilon_{i} \tau \sigma\left(Z_{i} \backslash\{1\}, Z_{i-1}\right) \varepsilon_{i-1}, \varepsilon_{j-1} \sigma\left(Z_{j-1}, Z_{j} \backslash\{2\}\right) \tau \varepsilon_{j} \mid 2 \leqslant i, j \leqslant 4\right\}
$$

generates $\varepsilon K G \varepsilon$.

Proof. We know from 2.6 that $\varepsilon K G \varepsilon$ is generated by

$$
\bigcup_{I, J} \varepsilon\left(Z_{|I|}, I\right) \mathcal{G} \varepsilon\left(J, Z_{|J|}\right)
$$

so it suffices to show that all nonzero elements of this set occur among the proposed generators. Just for this proof, write $i$ for $|I|$ and $j$ for $|J|$. We consider $\varepsilon\left(Z_{i}, I\right) S_{4} \varepsilon\left(J, Z_{j}\right), \varepsilon\left(Z_{i}, I\right) \mu_{\kappa} \varepsilon\left(J, Z_{j}\right)$ and $\varepsilon\left(Z_{i}, I\right) \tau \varepsilon\left(J, Z_{j}\right)$ in turn.

Firstly:

$$
\varepsilon\left(Z_{i}, I\right) S_{4} \varepsilon\left(J, Z_{j}\right)=\varepsilon_{i} \sigma\left(Z_{i}, I\right) S_{4} \sigma\left(J, Z_{j}\right) \varepsilon_{j}=\varepsilon_{i} S_{4} \varepsilon_{j}
$$

Let $\sigma \in S_{4}$ and $\varepsilon_{i} \sigma \varepsilon_{j} \neq 0$. As $\varepsilon_{i} \sigma \varepsilon_{j}=\sigma \varepsilon_{M} \varepsilon_{j}$ with $M=Z_{i} \sigma$, we must have $Z_{i} \sigma=Z_{j}$, so $j=i$ and $\sigma$ leaves $Z_{i}$ (setwise) fixed. Therefore it is possible to write $\sigma=\sigma^{\prime} \sigma^{\prime \prime}$ where $\sigma^{\prime}$ leaves each element of $Z_{i}$ fixed and $\sigma^{\prime \prime} \in S_{i}$. Now $\varepsilon_{i}$ is, by definition, a multiple of $\prod_{j>i} \partial_{j}$, and direct multiplication shows $\left(\Pi_{j>i} \partial_{j}\right) \sigma^{\prime}=\Pi_{j>i} \partial_{j}$ : thus $\varepsilon_{i} \sigma^{\prime}=\varepsilon_{i}$ and so $\varepsilon_{i} \sigma \varepsilon_{i}=\varepsilon_{i} \sigma^{\prime \prime} \varepsilon_{i}$. This proves that $\varepsilon_{i} S_{4} \varepsilon_{j} \subseteq\{0\} \bigcup \varepsilon_{i} S_{i} \varepsilon_{i}$.

Secondly: observe that $\mu_{\kappa}$ commutes with each $\partial_{k}$ and hence also with each $\varepsilon_{I}$, and with all permutations that fix 1 . Thus

$$
\begin{aligned}
\varepsilon\left(Z_{i}, I\right) \mu_{\kappa} \varepsilon\left(J, Z_{j}\right) & =\varepsilon_{i} \sigma\left(Z_{i}, I\right) \mu_{\kappa} \varepsilon_{J} \sigma\left(J, Z_{j}\right) \\
& =\sigma\left(Z_{i}, I\right) \mu_{\kappa} \varepsilon_{I} \varepsilon_{J} \sigma\left(J, Z_{j}\right)
\end{aligned}
$$

and this is zero unless $I=J$. Now if $1 \notin I$ then $\partial_{1} \mu_{\kappa}=\partial_{1}$ yields that $\varepsilon_{l} \mu_{\kappa}=\varepsilon_{I}$, and so

$$
\begin{aligned}
\varepsilon\left(Z_{i}, I\right) \mu_{\kappa} \varepsilon\left(I, Z_{i}\right) & =\sigma\left(Z_{i}, I\right) \varepsilon_{I} \mu_{\kappa} \varepsilon\left(I, Z_{i}\right) \\
& =\varepsilon\left(Z_{i}, I\right) \varepsilon\left(I, Z_{i}\right)=\varepsilon_{i} \in \varepsilon_{i} S_{i} \varepsilon_{i} .
\end{aligned}
$$

On the other hand, if $1 \in I$ then $\sigma\left(I, Z_{i}\right)$ fixes 1 and so commutes with $\mu_{\kappa}$ : hence

$$
\varepsilon\left(Z_{i}, I\right) \mu_{\kappa} \varepsilon\left(I, Z_{i}\right)=\varepsilon_{i} \sigma\left(Z_{i}, I\right) \mu_{\kappa} \sigma\left(I, Z_{i}\right) \varepsilon_{i}=\varepsilon_{i} \mu_{\kappa} \varepsilon_{i} .
$$

Thirdly: observe that $\tau$ commutes with all permutations that fix 1 and 2 and also with $\partial_{3}$ and $\partial_{4}$. Moreover, $\partial_{1} \tau=\partial_{1}, \tau \partial_{2}=\partial_{2}$ and $\partial_{2} \tau \partial_{1}=\partial_{2} \sigma_{2} \partial_{1}$ (recall that $\sigma_{2}$ is the permutation which interchanges 1 and 2 and fixes 3 and 4).

Writing $\varepsilon\left(Z_{i}, I\right) \tau \varepsilon\left(J, Z_{j}\right)$ in the form $\sigma\left(Z_{i}, I\right) \varepsilon_{I} \tau \varepsilon_{J} \sigma\left(J, Z_{j}\right)$ we see that if $1 \notin I$ then $\varepsilon_{I}$ has a factor $\partial_{1}$ so $\tau$ is redundant; if $2 \notin J$ then $\varepsilon_{J}$ has a factor $\partial_{2}$ so $\tau$ is again redundant; if $2 \notin I$ and $1 \notin J$ then $\varepsilon_{I} \tau \varepsilon_{J}$ has a factor $\partial_{2} \tau \partial_{1}$ so this generator belongs to $\varepsilon\left(Z_{i}, I\right) S_{4} \varepsilon\left(J, Z_{j}\right)$; and finally if $I \backslash\{1,2\} \neq J \backslash\{1,2\}$ then $\varepsilon_{I} \tau \varepsilon_{J}$ has a factor $\left(1-\partial_{3}\right) \partial_{3}$ or $\left(1-\partial_{4}\right) \partial_{4}$ and so is zero. 
Thus the following cases remain for consideration:

(i) $\{1,2\} \subseteq I=J$,

(ii) $\{1,2\} \subseteq I$ and $J=I \backslash\{1\}$,

(iii) $\{1,2\} \subseteq J$ and $I=J \backslash\{2\}$.

In cases (i) and (ii), $\sigma\left(Z_{i}, I\right)$ fixes 1 and 2 and hence commutes with $\tau$, so we have

$$
\varepsilon\left(Z_{i}, I\right) \tau \varepsilon\left(J, Z_{j}\right)=\varepsilon_{i} \tau \sigma\left(Z_{i}, I\right) \sigma\left(I, Z_{i}\right) \varepsilon_{i}=\varepsilon_{i} \tau \varepsilon_{i}
$$

and

$$
\begin{aligned}
\varepsilon\left(Z_{i}, I\right) \tau \varepsilon\left(J, Z_{j}\right) & =\varepsilon_{i} \tau \sigma\left(Z_{i}, I\right) \sigma\left(I \backslash\{1\}, Z_{i-1}\right) \varepsilon_{i-1} \\
& =\varepsilon_{i} \tau \sigma\left(Z_{i} \backslash\{1\}, Z_{i-1}\right) \varepsilon_{i-1},
\end{aligned}
$$

respectively.

In case (iii), $\sigma\left(J, Z_{j}\right)$ commutes with $\tau$ so we have

$$
\begin{aligned}
\varepsilon\left(Z_{i}, I\right) \tau \varepsilon\left(J, Z_{j}\right) & =\varepsilon_{j-1} \sigma\left(Z_{j-1}, J \backslash\{2\}\right) \sigma\left(J, Z_{j}\right) \tau \varepsilon_{j} \\
& =\varepsilon_{j-1} \sigma\left(Z_{j-1}, Z_{j} \backslash\{2\}\right) \tau \varepsilon_{j} .
\end{aligned}
$$

(The products of the permutations in these last two calculations are tedious but straightforward consequences of the definitions.) This completes the proof of the proposition.

The symbols $\varepsilon_{i} \tau \sigma\left(Z_{i} \backslash\{1\}, Z_{i-1}\right) \varepsilon_{i-1}$ and $\varepsilon_{j-1} \sigma\left(Z_{j-1}, Z_{j} \backslash\{2\}\right) \tau \varepsilon_{j}$ are rather unwieldy so we shall abbreviate them to $(i \rightarrow i-1)$ and $(j-1 \rightarrow j)$, respectively. Also note that $\varepsilon_{1} S_{1} \varepsilon_{1}=\left\{\varepsilon_{1} \mu_{1} \varepsilon_{1}\right\}$, while $\varepsilon_{i} S_{i} \varepsilon_{i}$ is generated by $\varepsilon_{i} \sigma_{2} \varepsilon_{i}$ and $\varepsilon_{i} \sigma_{i} \varepsilon_{i}$ when $i=2,3$ or 4 . Thus we have the following.

\subsection{Corollary. The union of}

$$
\left\{\varepsilon_{i} \sigma_{2} \varepsilon_{i}, \varepsilon_{i} \sigma_{i} \varepsilon_{i},(i-1 \rightarrow i),(i \rightarrow i-1) \mid 2 \leqslant i \leqslant 4\right\}
$$

and

$$
\left\{\varepsilon_{i} \mu_{\kappa} \varepsilon_{i}, \varepsilon_{i} \tau \varepsilon_{i} \mid 1 \leqslant i \leqslant 4, \kappa \in Z\right\}
$$

generates $\varepsilon K G \varepsilon$.

Further applications of 2.1 become possible when 3 is a unit in $K$, for then it is known that $K S_{3} \simeq K S_{2} \oplus \operatorname{Mat}(2, K)$ and

$$
\frac{1}{3}\left(1+\sigma_{3}+\sigma_{3}^{2}\right), \quad \frac{1}{3}\left(1-\sigma_{3}+\sigma_{2} \sigma_{3}-\sigma_{2} \sigma_{3}^{2}\right), \quad \frac{1}{3}\left(1-\sigma_{3}^{2}-\sigma_{2} \sigma_{3}+\sigma_{2} \sigma_{3}^{2}\right)
$$

are pairwise orthogonal idempotents with sum 1 . (This is readily verified by direct calculation; see also the description in Boerner (1963) of Young's "natural representation" of $S_{n}$.) We know from 3.2 that $\varepsilon_{3}$ and $\varepsilon_{4}$ commute with $S_{3}$ and so 
$r \rightarrow \varepsilon_{i} r \varepsilon_{i}(i=3,4)$ define ring homomorphisms $K S_{3} \rightarrow \varepsilon_{i} K G \varepsilon_{i}$. Thus $\varepsilon K G \varepsilon$ contains the direct sum $\varepsilon_{3} K S_{3} \varepsilon_{3} \oplus \varepsilon_{4} K S_{3} \varepsilon_{4}$ of the homomorphic images $\varepsilon_{i} K S_{3} \varepsilon_{i}$ of $K S_{2} \oplus \operatorname{Mat}(2, K)$. In particular, we obtain 6 pairwise orthogonal idempotents with sum $\varepsilon_{3}+\varepsilon_{4}$.

All this is available once we focus our attention on $Z_{(2)} G$ instead of the general $K G$, but a lot more will be needed. There is one more step which can be sketched before we begin investigating the action of $Z_{(2)} G$ on particular modules. This exploits the fact that, as we hinted before, $\varepsilon_{1}$ will annihilate all the modules we look at, so they may be viewed not only as $Z_{(2)} G$ or $\varepsilon Z_{(2)} G \varepsilon$ modules, respectively, but also as modules for the quotients of these rings modulo their (two-sided) ideals generated by $\varepsilon_{1}$. Now the definitions of $\varepsilon_{1}$ and $\varepsilon_{2}$ give

$$
\varepsilon_{2}=\left(-\varepsilon_{1}-\sigma_{2} \varepsilon_{1} \sigma_{2}\right)+\partial_{3} \partial_{4},
$$

and we have already noted that $\partial_{3} \partial_{4}$ commutes with $\mu_{-1}, \sigma_{2}$ and $\tau$. The semigroup $H$ generated by $\sigma_{2}$ and $\sigma_{2} \mu_{-1} \tau$ is isomorphic to $S_{3}$. Thus

$$
Z_{(2)} S_{2} \oplus \operatorname{Mat}\left(2, Z_{(2)}\right) \simeq Z_{(2)} H \rightarrow Z_{(2)} G / Z_{(2)} G \varepsilon_{1} Z_{(2)} G
$$

defined by $r \mapsto \varepsilon_{2} r \varepsilon_{2}+Z_{(2)} G \varepsilon_{1} Z_{(2)} G$ is a ring homomorphism, and so is $Z_{(2)} H \rightarrow$ $\varepsilon Z_{(2)} G \varepsilon / \varepsilon Z_{(2)} G \varepsilon_{1} Z_{(2)} G \varepsilon, r \mapsto \varepsilon_{2} r \varepsilon_{2}+\varepsilon Z_{(2)} G \varepsilon_{1} Z_{(2)} G \varepsilon$. This prepares the way for yet another application of 2.1. Unfortunately we find it necessary to take one even more ad hoc step before we get through. That, and what one would obtain from this paragraph and the last, will be telescoped into a single, complex move. The purpose of these two paragraphs has been to offer at least some partial motivation for what might otherwise appear a set of quite arbitrary choices, and to indicate the nature of some calculations which will be suppressed.

\section{Idempotents and further reduction}

In this section we look more specifically at the action of $\varepsilon Z_{(2)} G \varepsilon$ on $W \varepsilon$. From the definition of the $\partial_{i}$ it is immediate that each (Lie) monomial in $W$ is either annihilated or left unchanged when acted upon by a $\partial_{i}$; so the same can be said for the $1-\partial_{i}$ and hence also for the $\varepsilon_{i}$. In fact, a monomial must be annihilated by $\varepsilon_{i}$ unless it is the (Lie) product of precisely the first $i$ variables (in any order and any bracketing, repeated factors allowed): so that to each monomial there is at most one exceptional $\varepsilon_{i}$. (Note that $\varepsilon_{1}$ must annihilate every monomial of degree 4 so $W \varepsilon_{1}=0$ : this will be taken for granted without further reference.) It follows then that each monomial is either annihilated or fixed by $\varepsilon$. On examining the 60 basic monomials of degree 4 in turn, we find that 42 are annihilated by $\varepsilon$ and 18 are left unchanged; these 18 will then form a $Z_{(2)}$-basis for $W \varepsilon$. However 
we shall find another basis more convenient to use as our starting point. The reason for this is that while the diagonal generators $\mu_{\kappa}$ of $G$ act very simply on each monomial (multiplying it by $\kappa^{m}$ where $m$ is the degree of the monomial in $x$ ), the permutation matrices in $G$ mix basic and nonbasic monomials. This complication cannot be entirely avoided, but one can do better than by using basic monomials.

4.1. LEMMA. The following sets are bases for the $W \varepsilon_{i}$ :

$$
\begin{aligned}
& W \varepsilon_{2}:\{y x x y, y x x x, x y y y\}, \\
& W \varepsilon_{3}:\{y x x z, z x x y,(y x)(x z), z y y x, x y y z,(z y)(y x), x z z y, y z z x,(x z)(z y)\}, \\
& W \varepsilon_{4}:\{t x y z,(t x)(y z), t y z x,(t y)(z x), t z x y,(t z)(x y)\} .
\end{aligned}
$$

Proof. Recall that $W \varepsilon_{1}=0$ and, as $\varepsilon=\varepsilon_{1}+\varepsilon_{2}+\varepsilon_{3}+\varepsilon_{4}$ and the $\varepsilon_{i}$ are pairwise orthogonal $W \varepsilon=W \varepsilon_{2} \oplus W \varepsilon_{3} \oplus W \varepsilon_{4}$. The three sets listed above lie in the relevant $W_{\varepsilon}$, and their union has the right cardinality for a basis of $W_{\varepsilon}$ : this much is obvious. It is therefore sufficient to show that the union spans $W E$. A routine calculation shows that each of the 18 basics fixed by $\varepsilon$ is in the span of the union and thus completes the proof. Details are omitted.

With reference to the basis of $W \varepsilon$ just found we can identify End $Z_{Z_{(2)}} W \varepsilon$ with $\operatorname{Mat}\left(18, Z_{(2)}\right)$. Let $S$ stand for the homomorphic image in $\operatorname{End}_{Z_{(2)}} W \varepsilon$ of $\varepsilon Z_{(2)} G \varepsilon$. We forget $\varepsilon Z_{(2)} G \varepsilon$ for the time being, transferring the names of its elements to the corresponding elements of $S$. Thus for instance we have $\varepsilon_{1}=0$ and $\varepsilon=\varepsilon_{2}+\varepsilon_{3}+$ $\varepsilon_{4}=1$ in $S$ for this is how $\varepsilon_{1}$ and $\varepsilon$ act on $W \varepsilon$. Proposition 3.4 provides us with a generating set for $S$.

The aim of this section is, in effect, to understand precisely which elements of End $_{Z_{(2)}} W \varepsilon$ lie in $S$. The decisive step is that we can pick 12 pairwise orthogonal idempotents $\xi_{1}, \ldots, \xi_{12}$ in $S$, with sum 1 . These lead in turn to elements $\varepsilon_{i j}^{k}$ satisfying 2.3 and a further reduction of $S$ along the lines of Section 2.

One way of proceeding at this stage would be to give explicitly matrices describing the action of the known generating set of $S$ on our basis of $W \varepsilon$. However the matrices so obtained do not easily yield the required elements $\varepsilon_{i j}^{k}$, so we shall omit them on the grounds that they could easily be recovered from the matrices we do actually write down. Our first step is to change to a different basis in $W \varepsilon$ : we then give the action of $S$ on that basis. Define

$$
\begin{aligned}
& \mathbf{a}_{1}=y x x y+y x x x-x y y y, \\
& \mathbf{a}_{2}=2 y x x y-y x x x-2 x y y y, \\
& \mathbf{a}_{3}=2 y x x y+2 y x x x+x y y y, \\
& \mathbf{a}=-3 y x x z+z x x y+3(y x)(x z), \\
& \mathbf{a}_{4}=\mathbf{a}\left(2-\sigma_{3}-\sigma_{3}^{2}\right),
\end{aligned}
$$




$$
\begin{aligned}
& \mathbf{a}_{5}=\mathbf{a}\left(1-2 \sigma_{3}+\sigma_{3}^{2}\right), \\
& \mathbf{a}_{6}=\mathbf{a}\left(1+\sigma_{3}+\sigma_{3}^{2}\right), \\
& \mathbf{a}_{7}=y x x z, \\
& \mathbf{a}_{8}=(y x)(x z), \\
& \mathbf{a}_{9}=\mathbf{a}_{7} \sigma_{3}, \\
& \mathbf{a}_{10}=\mathbf{a}_{8} \sigma_{3}, \\
& \mathbf{a}_{11}=\mathbf{a}_{7} \sigma_{3}^{2}, \\
& \mathbf{a}_{12}=\mathbf{a}_{8} \sigma_{3}^{2}, \\
& \mathbf{a}_{13}=-(t x)(y z)-t y z x+(t y)(z x)+t z x y, \\
& \mathbf{a}_{14}=-2(t x)(y z)+(t y)(z x)+(t z)(x y), \\
& \mathbf{a}_{15}=-\mathbf{a}_{13} \sigma_{3}, \\
& \mathbf{a}_{16}=-\mathbf{a}_{14} \sigma_{3}, \\
& \mathbf{a}_{17}=t x y z+t y z x+t z x y, \\
& \mathbf{a}_{18}=(t x)(y z)+(t y)(z x)+(t z)(x y) .
\end{aligned}
$$

(The element a which appears fourth in this list is simply a useful piece of shorthand.)

4.2. LEMMA. The foilowing sets are bases for the $W \varepsilon_{i}$ :

$$
\begin{aligned}
& W \varepsilon_{2}:\left\{\mathbf{a}_{1}, \mathbf{a}_{2}, \mathbf{a}_{3}\right\}, \\
& W \varepsilon_{3}:\left\{\mathbf{a}_{4}, \ldots, \mathbf{a}_{12}\right\}, \\
& W \varepsilon_{4}:\left\{\mathbf{a}_{13}, \ldots, \mathbf{a}_{18}\right\} .
\end{aligned}
$$

PROOF. Each element of the proposed bases is expressed in terms of the basis elements of the corresponding $W \varepsilon_{i}$ which we already know from 4.1 . Thus it is sufficient to show that the matrices of coefficients expressing the $a_{j}$ in terms of those bases are invertible. The straightforward details are omitted.

Next the promised matrices expressing the action of $S$ on $W \varepsilon$ with reference to the basis of the $\mathbf{a}_{i}$. Where a matrix would have fractional entries, for simplicity we display a multiple (by a unit of $Z_{(2)}$ ). In each matrix the row labelled by $\mathbf{a}_{i}$ contains the coordinates of the image of $\mathbf{a}_{i}$. Thus, for example, the first row of the first matrix means that $\mathbf{a}_{1}\left(\varepsilon_{2} \sigma_{2} \varepsilon_{2}\right)=-\mathbf{a}_{1}$; the fourth row of the matrix for $3(3 \rightarrow 2)$ is blank because $\mathbf{a}_{7}(3 \rightarrow 2)=0$.

\subsection{Action on $W \varepsilon_{2}$.}

$\begin{array}{ccccc} & \mathbf{a}_{1} & \mathbf{a}_{2} & \mathbf{a}_{3} \\ \boldsymbol{\varepsilon}_{2} \boldsymbol{\sigma}_{2} \varepsilon_{2}: & \mathbf{a}_{1} & -1 & & \\ \mathbf{a}_{2} & & & -1 \\ \mathbf{a}_{3} & & -1 & \end{array}$




$$
\begin{array}{lllll} 
& & \multicolumn{1}{c}{\mathbf{a}_{1}} & \multicolumn{1}{c}{\mathbf{a}_{2}} & \multicolumn{1}{c}{\mathbf{a}_{3}} \\
3 \varepsilon_{2} \mu_{\kappa} \varepsilon_{2}: & \mathbf{a}_{1} & \kappa\left(2 \kappa^{2}-\kappa+2\right) & -\kappa^{2}(\kappa-1) & \kappa(\kappa-1) \\
& \mathbf{a}_{2} & -2(\kappa+2) \kappa(\kappa-1) & \kappa^{2}(\kappa+2) & 2 \kappa(\kappa-1) \\
& \mathbf{a}_{3} & 2 \kappa(\kappa-1)(2 \kappa+1) & -2 \kappa^{2}(\kappa-1) & \kappa(2 \kappa+1)
\end{array}
$$

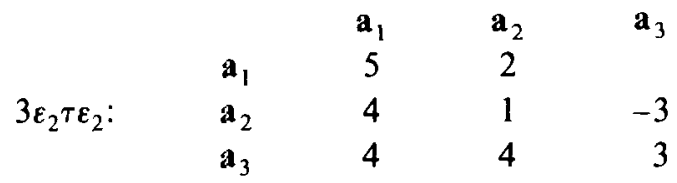

$3(2 \rightarrow 3):$

$\begin{array}{rrrrrrrrrr} & \mathbf{a}_{4} & \mathbf{a}_{5} & \mathbf{a}_{6} & \mathbf{a}_{7} & \mathbf{a}_{8} & \mathbf{a}_{9} & \mathbf{a}_{10} & \mathbf{a}_{11} & \mathbf{a}_{12} \\ \mathbf{a}_{1} & 4 & & 1 & 24 & -18 & & & -12 & 9 \\ \mathbf{a}_{2} & -1 & -3 & -4 & -24 & 18 & & & -24 & 18 \\ \mathbf{a}_{3} & 8 & & 2 & 48 & -36 & & & -24 & 18\end{array}$

4.4. Action on $W \varepsilon_{3}$.

$3 \varepsilon_{3} \sigma_{2} \varepsilon_{3}$

$\begin{array}{lrrrrrrrrr} & \mathbf{a}_{4} & \mathbf{a}_{5} & \mathbf{a}_{6} & \mathbf{a}_{7} & \mathbf{a}_{8} & \mathbf{a}_{9} & \mathbf{a}_{10} & \mathbf{a}_{11} & \mathbf{a}_{12} \\ \mathbf{a}_{4} & & 9 & & 24 & -18 & -48 & 36 & 24 & -18 \\ \mathbf{a}_{5} & 9 & & & 48 & -36 & -24 & 18 & -24 & 18 \\ \mathbf{a}_{6} & & & -9 & -24 & 18 & -24 & 18 & -24 & 18 \\ \mathbf{a}_{7} & & -1 & 1 & & & 9 & -9 & & \\ \mathbf{a}_{8} & & & & & & & & -3 & \\ \mathbf{a}_{9} & 1 & & 1 & 9 & -9 & & & & \\ \mathbf{a}_{10} & & & & & -3 & & & & \\ \mathbf{a}_{11} & -1 & 1 & 1 & & & & & & -3 \\ \mathbf{a}_{12} & & & & & & & & & \end{array}$


$\varepsilon_{3} \sigma_{3} \varepsilon_{3}$

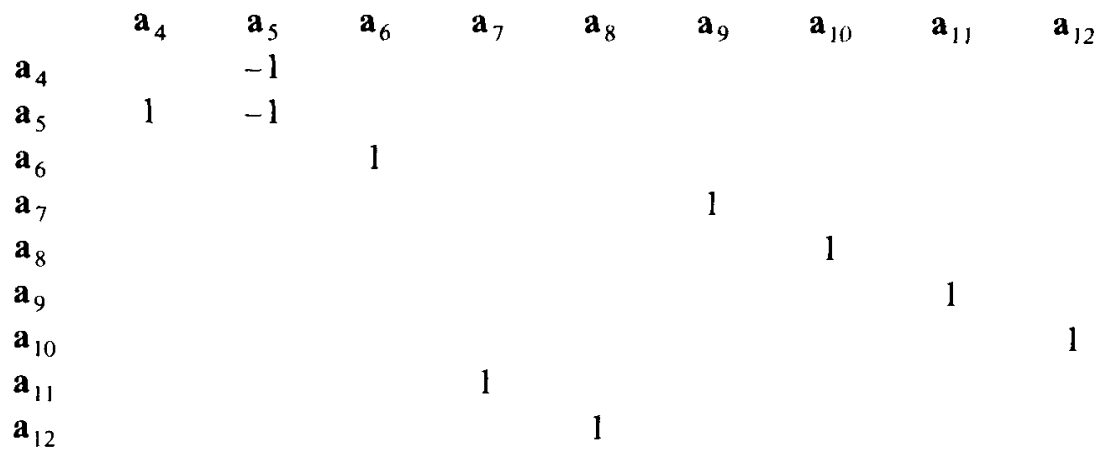

$3 \varepsilon_{3} \mu_{\kappa} \varepsilon_{3}$

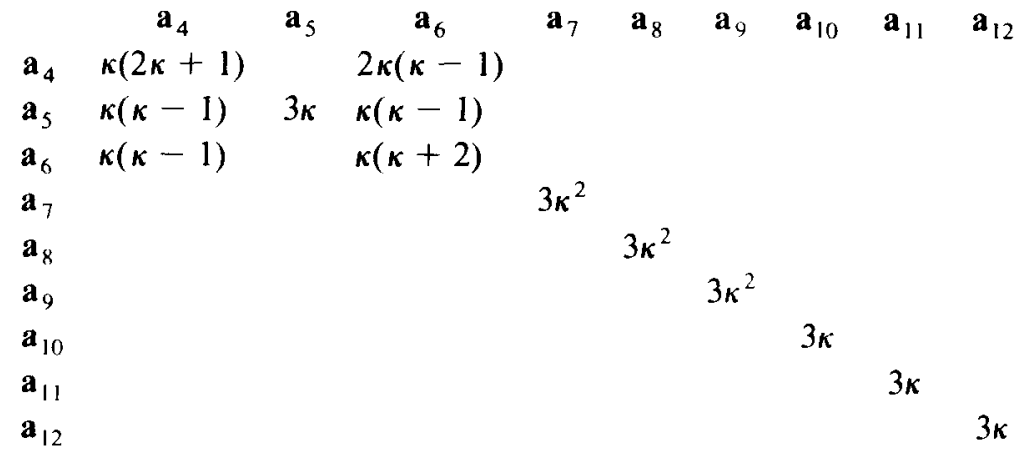

$3 \varepsilon_{3} \tau \varepsilon_{3}$

$\begin{array}{lrrrrrrrrr} & \mathbf{a}_{4} & \mathbf{a}_{5} & \mathbf{a}_{6} & \mathbf{a}_{7} & \mathbf{a}_{8} & \mathbf{a}_{9} & \mathbf{a}_{10} & \mathbf{a}_{11} & \mathbf{a}_{12} \\ \mathbf{a}_{4} & 3 & -4 & 4 & & & 48 & -36 & & \\ \mathbf{a}_{5} & & 1 & 2 & & & 24 & -18 & \\ \mathbf{a}_{6} & & -2 & 5 & & & 24 & -18 & \\ \mathbf{a}_{7} & 1 & -1 & 3 & & -9 & 9 & \\ \mathbf{a}_{8} & & & & & 3 & & 3 & \\ \mathbf{a}_{9} & & & & & & 3 & & 3 & \\ \mathbf{a}_{10} & & & & & & & & & 3 \\ \mathbf{a}_{11} & & & & & & & & & \end{array}$




$$
\begin{aligned}
& \begin{array}{llll}
\mathbf{a}_{1} & \mathbf{a}_{2} & \mathbf{a}_{3}
\end{array} \\
& \begin{array}{rrrr}
a_{4} & 12 & -7 & -2 \\
a_{5} & 12 & -5 & 2
\end{array} \\
& \begin{array}{llll}
a_{6} & -6 & 4 & 2
\end{array} \\
& 3(3 \rightarrow 2): \quad \begin{array}{l}
\mathbf{a}_{7} \\
\mathbf{a}_{8}
\end{array} \\
& \begin{array}{lll}
\mathbf{a}_{9} & 2 & -1
\end{array} \\
& a_{10} \\
& \begin{array}{llll}
\mathbf{a}_{11} & 1 & -1 & -1
\end{array} \\
& a_{12}
\end{aligned}
$$

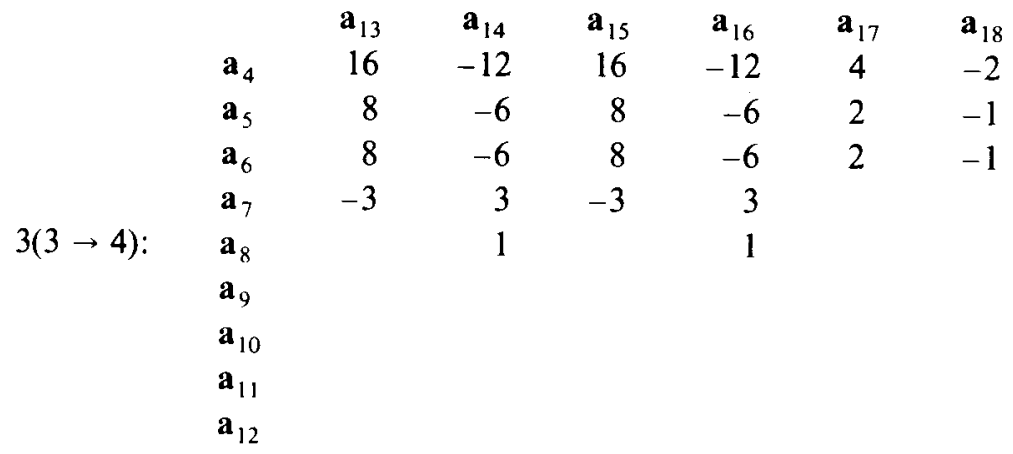

4.5. Action on $W \varepsilon_{4}$.

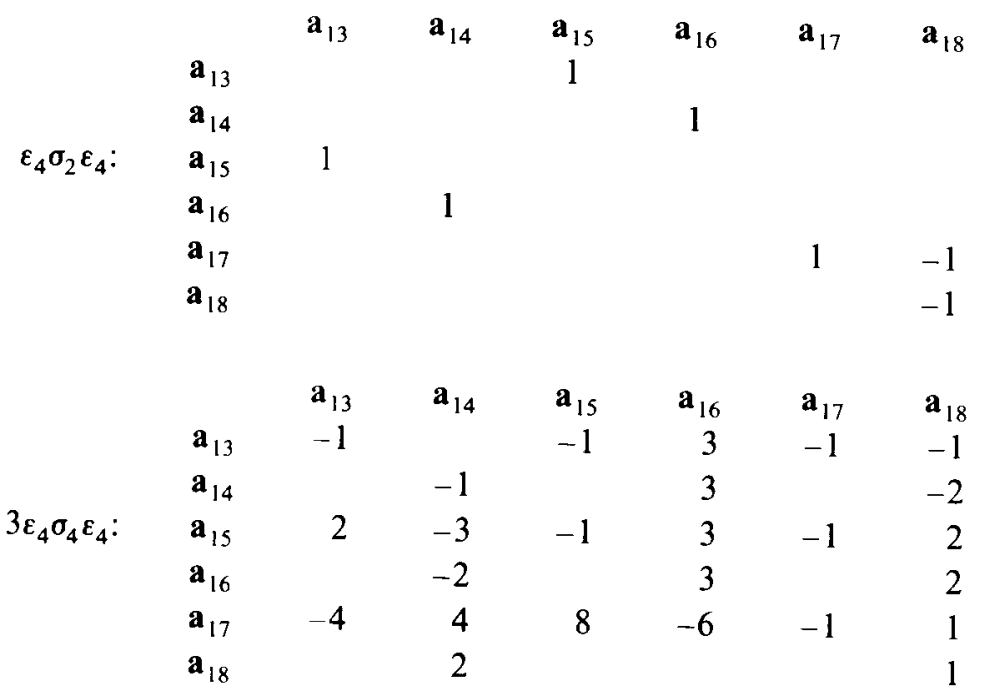




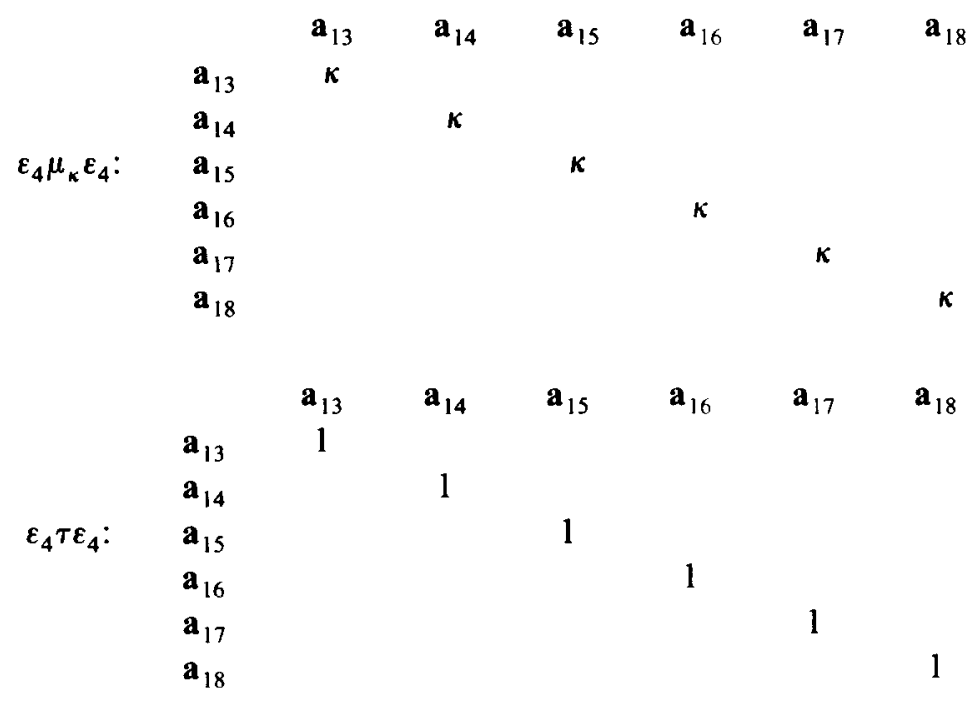

$(4 \rightarrow 3):$
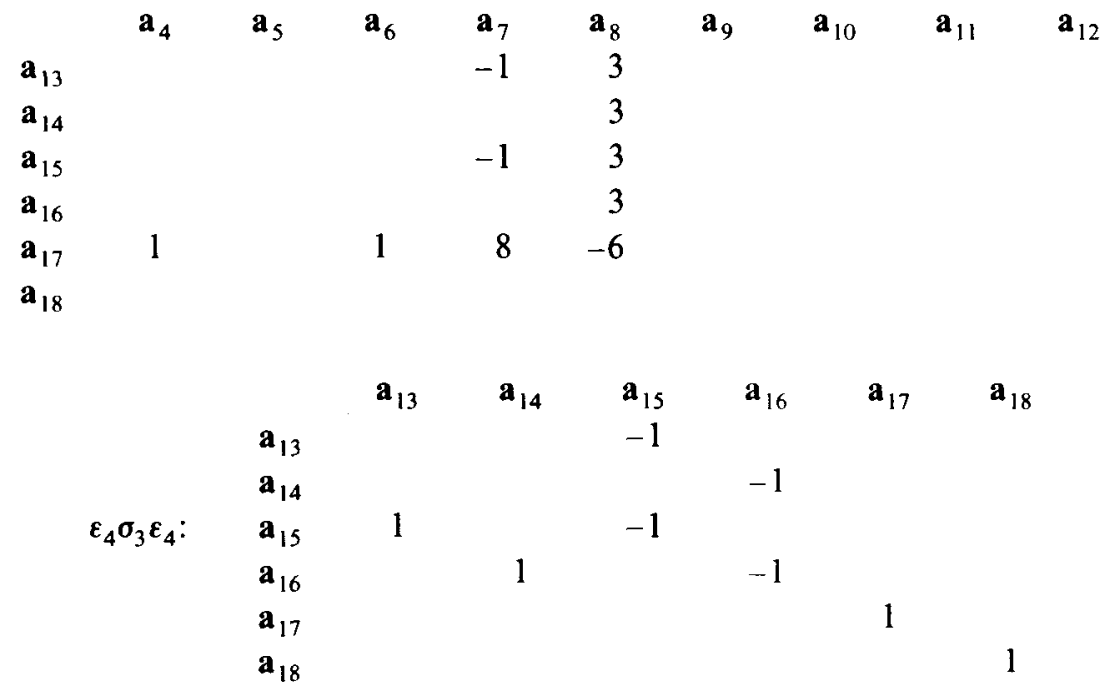

[This last matrix is only included as an aid to the calculation of $\xi_{10}, \xi_{11}$ and $\xi_{12}$ later in this section.]

We proceed now to pick the $\xi_{11} \cdots \xi_{12}$. A little shorthand will help. Let $f$ be a polynomial over $Z_{(2)}$. We abbreviate $f(\varphi \alpha \varphi, \varphi \beta \varphi, \ldots)$ to $\varphi \llbracket f(\alpha, \beta, \ldots) \rrbracket \varphi$ where the significance of the symbols $\varphi, \alpha, \beta, \ldots$ will be clear from the context. Thus for 
example, $\varepsilon_{3} \llbracket \sigma_{2}(\tau+1) \rrbracket \varepsilon_{3}$ means $\left(\varepsilon_{3} \sigma_{2} \varepsilon_{3}\right)\left(\varepsilon_{3} \tau \varepsilon_{3}+\varepsilon_{3}\right)$. Define

$$
\begin{aligned}
& \zeta=\varepsilon_{2} \llbracket \sigma_{2} \mu_{-1} \tau \rrbracket \varepsilon_{2}, \\
& \eta=\varepsilon_{3} \llbracket\left(1+\left(\sigma_{2}+\tau-1\right) \sigma_{3}^{2}\right)(1-\tau) \sigma_{2} \rrbracket \varepsilon_{3} .
\end{aligned}
$$

The last paragraph of Section 3 shows how $\zeta$ leads to

$$
\begin{aligned}
& \xi_{1}=\frac{1}{3} \varepsilon_{2} \llbracket 1+\zeta+\zeta^{2} \rrbracket \varepsilon_{2}, \\
& \xi_{2}=\frac{1}{3} \varepsilon_{2} \llbracket 1-\zeta+\sigma_{2} \zeta-\sigma_{2} \zeta^{2} \rrbracket \varepsilon_{2}, \\
& \xi_{3}=\frac{1}{3} \varepsilon_{2} \llbracket 1-\zeta^{2}-\sigma_{2} \zeta+\sigma_{2} \zeta^{2} \rrbracket \varepsilon_{2}:
\end{aligned}
$$

three pairwise orthogonal idempotents with sum $\varepsilon_{2}$. Direct calculation shows that these act (on $\left.W \varepsilon_{2}\right)$ as $\operatorname{diag}(1,0,0), \operatorname{diag}(0,1,0)$ and $\operatorname{diag}(0,0,1)$ respectively. Next, use the matrices just given to calculate that on $W \varepsilon_{3}, \eta$ acts as $\operatorname{diag}(0,0,0,1,1,0,0,0,0)$ and that therefore $\xi_{7}, \xi_{8}$ and $\xi_{9}$ chosen as

$$
\begin{aligned}
& \xi_{7}=\eta, \\
& \xi_{8}=\varepsilon_{3} \llbracket \sigma_{3}^{2} \eta \sigma_{3} \rrbracket \varepsilon_{3}, \\
& \xi_{9}=\varepsilon_{3} \llbracket \sigma_{3} \eta \sigma_{3}^{2} \rrbracket \varepsilon_{3},
\end{aligned}
$$

act as pairwise orthogonal idempotents with the latter two acting as $\operatorname{diag}(0,0,0,0,0,1,1,0,0)$ and $\operatorname{diag}(0,0,0,0,0,0,0,1,1)$ respectively. Put

$$
\xi=\varepsilon_{3}-\left(\xi_{7}+\xi_{8}+\xi_{9}\right)
$$

note that $\xi \varepsilon_{3}=\varepsilon_{3} \xi=\xi ; \xi$ annihilates $\varepsilon_{2}$ and $\varepsilon_{4}$ and acts as $\operatorname{diag}(1,1,1,0, \ldots, 0)$ on $W \varepsilon_{3}$. One can readily see from the tables that multiplicatively $\frac{1}{3} \xi \sigma_{2} \xi\left(=\frac{1}{3} \xi \varepsilon_{3} \sigma_{2} \varepsilon_{3} \xi\right)$ and $\xi \sigma_{3} \xi\left(=\xi \varepsilon_{3} \sigma_{3} \varepsilon_{3} \xi\right)$ generate another isomorphic copy of the symmetric group $S_{3}$; hence

$$
\begin{aligned}
& \xi_{4}=\frac{1}{3} \xi \llbracket 1-\sigma_{3}+\frac{1}{3} \sigma_{2} \sigma_{3}-\frac{1}{3} \sigma_{2} \sigma_{3}^{2} \rrbracket \xi, \\
& \xi_{5}=\frac{1}{3} \xi \llbracket 1-\sigma_{3}^{2}-\frac{1}{3} \sigma_{2} \sigma_{3}+\frac{1}{3} \sigma_{2} \sigma_{3}^{2} \rrbracket \xi, \\
& \xi_{6}=\frac{1}{3} \xi \llbracket 1+\sigma_{3}+\sigma_{3}^{2} \rrbracket \xi,
\end{aligned}
$$

define three more pairwise orthogonal idempotents with sum $\xi$. These are seen by direct calculation to act as $\operatorname{diag}(1,0, \ldots, 0), \operatorname{diag}(0,1,0, \ldots, 0)$ and $\operatorname{diag}(0,0,1,0, \ldots, 0)$ respectively, and we now have $\xi_{4}+\cdots+\xi_{9}=\varepsilon_{3}$. Finally, put

$$
\begin{aligned}
& \xi_{10}=\frac{1}{3} \varepsilon_{4} \llbracket 1-\sigma_{3}+\sigma_{2} \sigma_{3}-\sigma_{2} \sigma_{3}^{2} \rrbracket \varepsilon_{4}, \\
& \xi_{11}=\frac{1}{3} \varepsilon_{4} \llbracket 1-\sigma_{3}^{2}-\sigma_{2} \sigma_{3}+\sigma_{2} \sigma_{3}^{2} \rrbracket \varepsilon_{4}, \\
& \xi_{12}=\frac{1}{3} \varepsilon_{4} \llbracket 1+\sigma_{3}+\sigma_{3}^{2} \rrbracket \varepsilon_{4}:
\end{aligned}
$$

we have seen in the second last paragraph of Section 3 that these are pairwise orthogonal idempotents with sum $\varepsilon_{4}$. Again by direct calculation these may be 
shown to act (on $W \varepsilon_{4}$ ) as $\operatorname{diag}(1,1,0,0,0,0), \operatorname{diag}(0,0,1,1,0,0)$ and $\operatorname{diag}(0,0,0,0,1,1)$ respectively. As $\varepsilon_{2}+\varepsilon_{3}+\varepsilon_{4}=1$ all this combined proves the following.

4.6. LeMMA. The $\xi_{1}, \ldots, \xi_{12}$ so defined are pairwise orthogonal idempotents with sum 1 in $S$.

It will now be clear why our basis of $W \varepsilon$ was so chosen: the idempotents $\xi_{i}$ all act diagonally on it. Recall that $e_{n}(i, j)$ is the $n \times n$ elementary matrix whose entries are all 0 apart from the $(i, j)$ entry which is 1 .

4.7. LemMa. The following elements are in $S$.

$$
\begin{aligned}
& e_{18}(i, j), \quad i, j \in\{2,3,4,5\}, \\
& e_{18}(i, j)+e_{18}(i+1, j+1), \quad i, j \in\{7,9,11,13,15\} .
\end{aligned}
$$

Proof. Note that $e_{18}(2,3)=-\xi_{2} \varepsilon_{2} \sigma_{2} \varepsilon_{2} \xi_{3} \in S$ simply because the $(2,3)$ entry of the matrix for $\varepsilon_{2} \sigma_{2} \varepsilon_{2}$ is -1 . Also $e_{18}(7,9)+e_{18}(8,10)=\xi_{7} \varepsilon_{3} \sigma_{3} \varepsilon_{3} \xi_{8}$ is in $S$ because the $(7,9)$ and $(8,10)$ entries of $\varepsilon_{3} \sigma_{3} \varepsilon_{3}$ are 1 while the $(7,10),(8,9)$ entries are 0.

Similarly we find that $S$ contains

$$
\begin{array}{ll}
e_{18}(3,2) & \text { using } \varepsilon_{2} \sigma_{2} \varepsilon_{2}, \\
e_{18}(2,4), e_{18}(2,5) & \text { using }(2 \rightarrow 3), \\
e_{18}(4,2), e_{18}(5,2) & \text { using }(3 \rightarrow 2), \\
e_{18}(9,11)+e_{18}(10,12) & \text { using } \varepsilon_{3} \sigma_{3} \varepsilon_{3}, \\
e_{18}(11,7)+e_{18}(12,8) & \text { using } \varepsilon_{3} \sigma_{3} \varepsilon_{3}, \\
e_{18}(13,15)+e_{18}(14,16) & \text { using } \varepsilon_{4} \sigma_{2} \varepsilon_{4}, \\
e_{18}(15,13)+e_{18}(16,14) & \text { using } \varepsilon_{4} \sigma_{2} \varepsilon_{4}, \\
e_{18}(7,15)+e_{18}(8,16) & \text { using } 3\left[\xi_{7}(3 \rightarrow 4) \xi_{11}\right]\left[\xi_{10} \varepsilon_{4} \sigma_{4} \varepsilon_{4} \xi_{10}\right], \\
e_{18}(15,7)+e_{18}(16,8) & \text { using }\left[\xi_{11}(4 \rightarrow 3) \xi_{7}\right]\left[\xi_{7}\left(\varepsilon_{3} \sigma_{3} \varepsilon_{3}\right)\left(\varepsilon_{3} \sigma_{2} \varepsilon_{3}\right) \xi_{7}\right] .
\end{array}
$$

(Note that in each of the last two cases the given matrix has precisely one nonzero $2 \times 2$ submatrix.) The other $e_{18}(i, j)$ and $e_{18}(i, j)+e_{18}(i+1, j+1)$ are obtained from these by multiplication. This completes the proof of the lemma.

Rename

$$
\begin{aligned}
& e_{18}(1,1)=\varepsilon_{11}^{1}, \\
& e_{18}(i, j)=\varepsilon_{i-1, j-1}^{2} \quad \text { for } i, j \in\{2,3,4,5\}, \\
& e_{18}(6,6)=\varepsilon_{11}^{3}, \\
& e_{18}(i, j)+e_{18}(1+1, j+1)=\varepsilon_{(i-5) / 2,(j-5) / 2}^{4} \text { for } i, j \in\{7,9,11,13,15\}, \\
& e_{18}(17,17)+e_{18}(18,18)=\varepsilon_{11}^{5} .
\end{aligned}
$$


These elements satisfy 2.3 and 2.4 so it follows that

$$
e=\xi_{1}+\xi_{2}+\xi_{6}+\xi_{7}+\xi_{12}
$$

defines an idempotent $e$ with $S e S=S$ and 2.1, 2.2, 2.6 and 2.7 become applicable. Thus we can shift our attention to the ring $e S e$ and the module Wee. Write $\mathbf{w}_{1}=\mathbf{a}_{1}, \mathbf{w}_{2}=\mathbf{a}_{2}, \mathbf{w}_{3}=\mathbf{a}_{6}, \mathbf{w}_{4}=\mathbf{a}_{7}, \mathbf{w}_{5}=\mathbf{a}_{8}, \mathbf{w}_{6}=\mathbf{a}_{17}$ and $\mathbf{w}_{7}=\mathbf{a}_{18}$. Then $w_{1}, \ldots, w_{7}$ form a $Z_{(2)}$-basis for Wee. We identify $\operatorname{End}_{Z_{(2)}}$ Wee with $\operatorname{Mat}\left(7, Z_{(2)}\right)$ according to this basis.

Let $T$ stand for the image of $e S e$, under restriction to $W \varepsilon e$, in $\operatorname{Mat}\left(7, Z_{(2)}\right)$. This change from eSe to $T$ is essentially a notational matter: if an element of $e S e$ is written as $\rho=(\rho(i, j))$ according to $e S e \leqslant S \leqslant \operatorname{Mat}\left(18, Z_{(2)}\right)$, then the entries $\rho(i, j)$ of $\rho$ vanish unless $i, j \in\{1,2,6,7,8,17,18\}$; to obtain the corresponding element of $T$, we simply omit the rows and columns which must vanish because we started with an element of $e S e$. With this final shift of view our task becomes the study of $T$ and the $T$-submodules of $W \varepsilon e$.

The main result of this section gives a precise description of $T$ as subalgebra of $\operatorname{Mat}\left(7, Z_{(2)}\right)$. In order to state it, we make the following definitions.

$$
\alpha=(\alpha(i, j))=\left(\begin{array}{ccccc}
1 & 2 & 1 & \alpha_{1} & \alpha_{2} \\
4 & 1 & 2 & \alpha_{3} & \alpha_{4} \\
2 & 2 & 1 & \alpha_{5} & \alpha_{6} \\
1 & 1 & 1 & & \\
0 & 0 & 0 & & \\
2 & 1 & 1 & & \alpha_{8} \\
0 & 0 & 0 & & \alpha_{10}
\end{array}\right)
$$

where $\alpha_{1}, \ldots, \alpha_{6}$ are $1 \times 2$ and $\alpha_{7}, \ldots, \alpha_{10}$ are $2 \times 2$ submatrices;

$$
\begin{aligned}
& A_{1}=\left\{\kappa(4-3) \mid \kappa \in Z_{(2)}\right\} ; \\
& A_{2}=\left\{\kappa(2-1) \mid \kappa \in Z_{(2)}\right\} ; \\
& A_{3}=A_{5}=2 A_{1} ; \\
& A_{4}=A_{6}=A_{2} ; \\
& A_{7}=\left\{\kappa_{1}\left(\begin{array}{ll}
3 & -3 \\
0 & -1
\end{array}\right)+\kappa_{2}\left(\begin{array}{cc}
1 & 0 \\
0 & 1
\end{array}\right) \mid \kappa_{1}, \kappa_{2} \in Z_{(2)}\right\} ; \\
& A_{8}=\left\{\kappa_{1}\left(\begin{array}{ll}
1 & 1 \\
0 & 2
\end{array}\right)+\kappa_{2}\left(\begin{array}{cc}
-1 & 2 \\
0 & 2
\end{array}\right) \mid \kappa_{1}, \kappa_{2} \in Z_{(2)}\right\} ; \\
& A_{9}=\left\{\kappa_{1}\left(\begin{array}{cc}
4 & -4 \\
0 & -2
\end{array}\right)+\kappa_{2}\left(\begin{array}{cc}
8 & -6 \\
0 & 0
\end{array}\right) \mid \kappa_{1}, \kappa_{2} \in Z_{(2)}\right\} ; \\
& A_{10}=\left\{\kappa_{1}\left(\begin{array}{ll}
1 & -1 \\
0 & -1
\end{array}\right)+\kappa_{2}\left(\begin{array}{cc}
1 & 0 \\
0 & 1
\end{array}\right) \mid \kappa_{1}, \kappa_{2} \in Z_{(2)}\right\} .
\end{aligned}
$$


Let $T^{\prime}$ be the subset of $\operatorname{Mat}\left(7, Z_{(2)}\right)$ consisting of all matrices $\beta=(\beta(i, j))$ satisfying the following conditions:

$\beta(i, j) \in \alpha(i, j) Z_{(2)}$ for $j \leqslant 3$,

the $1 \times 2$ or $2 \times 2$ submatrix $\beta_{i}$ corresponding to $\alpha_{i}$ belongs to the set $A_{i}$ for $1 \leqslant i \leqslant 10$.

\subsection{THEOREM. $T=T^{\prime}$.}

Proof. It is straightforward to check that $T^{\prime}$ is a subalgebra of $\operatorname{Mat}\left(7, Z_{(2)}\right)$. Thus in order to prove that $T \leqslant T^{\prime}$ it is sufficient to show that $T^{\prime}$ contains the generating set of $T$ obtained by 2.6 from the known generating set of $S$. To see what this involves let us consider any generator of $S$ in the form of an element $\gamma$ of $\operatorname{Mat}\left(18, Z_{(2)}\right)$. The generators of eSe arise by premultiplying $\gamma$ by $\varepsilon_{1 j}^{l}$ and postmultiplying by $\varepsilon_{i 1}^{m}$ where $i, j, l, m$ have the appropriate ranges given by the definition of the $\varepsilon_{i j}^{k}$. Each (nontrivial) generator of $e S e$ so obtained has precisely one nonzero submatrix (of size $1 \times 1,1 \times 2,2 \times 1$ or $2 \times 2$ ) and the corresponding generator of $T$ is obtained by omitting the appropriate zero rows and columns to give a $7 \times 7$ matrix with precisely one nonzero submatrix. This can then be tested for inclusion in $T^{\prime}$. To verify this one must examine each submatrix of each of the matrices given in the tables 4.3 to 4.5 : a tedious but straightforward exercise.

For the converse we observe that $T^{\prime}$ has 29 generators each of which must be checked for inclusion in $T$. The following table lists for each generator of $T^{\prime}$, with two exceptions, an element of $S$ from which it may be obtained after pre- or postmultiplying, if necessary, by an appropriate element from those listed in 4.7.

$\begin{aligned} e_{7}(1,1) & \xi_{1} \\ 2 e_{7}(1,2) & \xi_{1}\left(\varepsilon_{2} \tau \varepsilon_{2}\right) \xi_{2} \\ e_{7}(1,3) & \xi_{1}(2 \rightarrow 3) \xi_{6} \\ 4 e_{7}(1,4)-3 e_{7}(1,5) & \xi_{1}(2 \rightarrow 3) \xi_{9} \\ 4 e_{7}(2,1) & \xi_{2}\left(\varepsilon_{2} \tau \varepsilon_{2}\right) \xi_{1} \\ e_{7}(2,2) & \xi_{2} \\ 2 e_{7}(2,3) & \xi_{3}(2 \rightarrow 3) \xi_{6} \\ 8 e_{7}(2,4)-6 e_{7}(2,5) & \xi_{2}(2 \rightarrow 3) \xi_{7} \\ 2 e_{7}(2,6)-e_{7}(2,7) & \xi_{5}(3 \rightarrow 4) \xi_{12} \\ 2 e_{7}(3,1) & \xi_{6}(3 \rightarrow 2) \xi_{1} \\ 2 e_{7}(3,2) & \xi_{6}(3 \rightarrow 2) \xi_{3} \\ e_{7}(3,3) & \xi_{6} \\ 8 e_{7}(3,4)-6 e_{7}(3,5) & \xi_{6}\left(\varepsilon_{3} \sigma_{2} \varepsilon_{3}\right) \xi_{7} \\ 2 e_{7}(3,6)-e_{7}(3,7) & \xi_{6}(3 \rightarrow 4) \xi_{12} \\ e_{7}(4,1) & \xi_{9}(3 \rightarrow 2) \xi_{1}\end{aligned}$




$\begin{array}{cl}e_{7}(4,2) & \xi_{9}(3 \rightarrow 2) \xi_{2} \\ e_{7}(4,3) & \xi_{7}\left(\varepsilon_{3} \tau \varepsilon_{3}\right) \xi_{6} \\ 3 e_{7}(4,4)-3 e_{7}(4,5)-e_{7}(5,5) & \xi_{7}\left(\varepsilon_{3} \sigma_{2} \varepsilon_{3}\right) \xi_{8} \\ e_{7}(4,4)+e_{7}(5,5) & \xi_{7} \\ e_{7}(4,6)+e_{7}(4,7)+2 e_{7}(5,7) & \xi_{10}\left(\varepsilon_{4} \sigma_{4} \varepsilon_{4}\right) \xi_{12} \\ e_{7}(4,6)-2 e_{7}(4,7)-2 e_{7}(5,7) & \xi_{11}\left(\varepsilon_{4} \sigma_{4} \varepsilon_{4}\right) \xi_{12} \\ e_{7}(6,2) & \xi_{12}(4 \rightarrow 3) \xi_{4} \\ e_{7}(6,3) & \xi_{12}(4 \rightarrow 3) \xi_{6} \\ 4 e_{7}(6,4)-4 e_{7}(6,5)-2 e_{7}(7,5) & \xi_{12}\left(\varepsilon_{4} \sigma_{4} \varepsilon_{4}\right) \xi_{10} \\ 8 e_{7}(6,4)-6 e_{7}(6,5) & \xi_{12}\left(\varepsilon_{4} \sigma_{4} \varepsilon_{4}\right) \xi_{11} \\ e_{7}(6,6)-e_{7}(6,7)-e_{7}(7,7) & \xi_{12}\left(\varepsilon_{4} \sigma_{4} \varepsilon_{4}\right) \xi_{12} \\ e_{7}(6,6)+e_{7}(7,7) & \xi_{12}\end{array}$

The exceptional cases are $2 e_{7}(1,6)-e_{7}(1,7)$ which is in $T$ because it is $e_{7}(1,3)\left[2 e_{7}(3,6)-e_{7}(3,7)\right]$ and $2 e_{7}(6,1)$ which is in $T$ because it is $e_{7}(6,3)\left[2 e_{7}(3,1)\right]$. This completes the proof of the theorem.

4.9. REMARK. This theorem could be used via a repeated application of 2.7 to provide a complete description of $Z_{(2)} G / \operatorname{Ann} W$. Thus the claim made in the introduction is justified, although it is clear that we have no need to perform this purely mechanical task.

We end this section with some consequences of Theorem 4.8.

4.10. Corollary. $W$ is generated as $Z_{(2)} G$-module by yxxz.

Proof. This follows via repeated application of 2.2 noting that $y x x z=\mathbf{a}_{7}=\mathbf{w}_{4}$ which can be seen by 4.8 to generate $W \varepsilon e$. (This result is related to 34.45 of Hanna Neumann (1967).)

It is very easy to check that $W_{\varepsilon} e$ has a submodule spanned as $Z_{(2)}$-module by $\left\{\mathbf{w}_{1}, \mathbf{w}_{2}, \mathbf{w}_{3}, 4 \mathbf{w}_{4}-3 \mathbf{w}_{5}, 2 \mathbf{w}_{6}-\mathbf{w}_{7}\right\}$. Since these elements are obviously independent modulo $2 W \varepsilon e$ we conclude that they form a $Z_{(2)}$-basis for their span and that that span is isolated as $Z_{(2)}$-submodule of $W \varepsilon e$. We denote by $U$ the corresponding submodule of $W$ and relabel as follows:

$$
\mathbf{u}_{1}=\mathbf{w}_{1}, \quad \mathbf{u}_{2}=\mathbf{w}_{2}, \quad \mathbf{u}_{3}=\mathbf{w}_{3}, \quad \mathbf{u}_{4}=4 \mathbf{w}_{4}-3 \mathbf{w}_{5}, \quad \mathbf{u}_{5}=2 \mathbf{w}_{6}-\mathbf{w}_{7} .
$$

Similarly $W \varepsilon e$ contains an isolated submodule with $Z_{(2)}$-basis $\left\{w_{5}, w_{7}\right\}$. Let $V$ be the corresponding submodule of $W$ and relabel $\mathbf{v}_{4}=w_{5}, \mathbf{v}_{5}=\mathbf{w}_{7}$. (It would of course be more natural to use subscripts 1,2 here instead of 4,5: the reason for 
our choice will become clear in Section 6.) Clearly $U \cap V=0$. By an easy application of 2.1, $U$ and $V$ are isolated as $Z_{(2)}$-submodules of $W$.

We may now use 4.8 to see that each submodule of $W \varepsilon e$ either is contained and has finite index in $U \varepsilon e$ or $V \varepsilon e$ or contains some nontrivial (2-power) multiple of each of them. It follows easily using 4.10 that each submodule not contained in one of the two isolated submodules we have found contains a nontrivial (2-power) multiple of $W \varepsilon e$. Such a submodule has finite index in $W \varepsilon e$ and is therefore not isolated unless it is $W \varepsilon e$ itself. This confirms another claim made in the introduction.

4.11. COROLlary. $U$ and $V$ are proper nontrivial isolated submodules of $W$ and they are the only such submodules.

We thus have $U \varepsilon e \oplus V \varepsilon e$ a submodule of finite index in $W \varepsilon e$ with $Z_{(2)}$-basis $\left\{\mathbf{u}_{1}, \ldots, \mathbf{u}_{5}, \mathbf{v}_{4}, \mathbf{v}_{5}\right\}$. In order to aid our discussion of submodules it is convenient to have the explicit form of the restriction $T_{U \oplus V}$ of $T$ to $U \varepsilon e \oplus V \varepsilon e$. We identify End $_{Z_{(2)}} U \varepsilon e \oplus$ End $_{Z_{(2)}} V \varepsilon e$ with $\operatorname{Mat}\left(5, Z_{(2)}\right) \oplus \operatorname{Mat}\left(2, Z_{(2)}\right)$ according to the bases consisting of the $\mathbf{u}_{i}$ and $\mathbf{v}_{i}$, and consider this direct sum naturally embedded in End $_{Z_{(2)}}(U \varepsilon e \oplus V \varepsilon e)$ which is in turn identified with $\operatorname{Mat}_{Z_{(2)}}\left(7, Z_{(2)}\right)$.

Define

$$
\boldsymbol{\alpha}^{\prime}=\left(\boldsymbol{\alpha}^{\prime}(i, j)\right)=\left(\begin{array}{lllll}
0 & 1 & 0 & 0 & 0 \\
2 & 0 & 1 & 1 & 0 \\
1 & 1 & 0 & 1 & 0 \\
2 & 2 & 2 & 0 & 1 \\
2 & 1 & 1 & 1 & 0
\end{array}\right)
$$

Let $T^{\prime \prime}$ be the set of all those $\gamma \oplus \delta$ in $\operatorname{Mat}\left(5, Z_{(2)}\right) \oplus \operatorname{Mat}\left(2, Z_{(2)}\right)$ which satisfy the following conditions:

$$
\begin{array}{ll}
\gamma(i, j) \in 2^{\alpha^{\prime}(i, j)} Z_{(2)} & \text { for all } i, j \text { in }\{1, \ldots, 5\} ; \\
\gamma(i, j) \equiv \delta(i, j)(\bmod 4) & \text { for }(i, j) \in\{(4,4),(5,4),(4,5)\} ; \\
\gamma(5,5) \equiv \delta(5,5)(\bmod 2) &
\end{array}
$$

(note that we have indexed rows and columns in the latter direct summand according to the subscripts on the $\mathbf{v}_{j}$ ).

\subsection{COROllary. $T_{U \oplus V}=T^{\prime \prime}$.}

Proof. This follows the same pattern as 4.8. First it is easy to check that $T^{\prime \prime}$ is a subalgebra. To prove $T_{U \oplus V} \leqslant T^{\prime \prime}$ it is therefore sufficient to show that each of the generators of $T_{U \oplus V}$ obtained from the known generating set of $T$ is contained in $T^{\prime \prime}$. Conversely, for each generator of $T^{\prime \prime}$ we must show there is an element of $T$ which acts like it on $U \varepsilon e \oplus V \varepsilon e$. The straightforward details are omitted. 
Now let $T_{U}$ and $T_{V}$ denote the restrictions to $U \varepsilon e$ and $V \varepsilon e$.

\subsection{Corollary.}

$$
\begin{aligned}
& T_{U}=\left\{\gamma \in \operatorname{Mat}\left(5, Z_{(2)}\right) \mid \gamma(i, j) \in 2^{\alpha^{\prime}(i, j)} Z_{(2)}\right\} \\
& T_{V}=\left\{\delta \in \operatorname{Mat}\left(2, Z_{(2)}\right) \mid \delta(i, j) \in 2^{\alpha^{\prime}(i, j)} Z_{(2)}\right\} .
\end{aligned}
$$

Proof. Only the second statement calls for comment. Comparing the moduli of the congruence conditions in the definition of $T^{\prime \prime}$ with the exponents $\alpha^{\prime}(i, j)$ for $i, j \in\{4,5\}$, one sees that $\gamma \oplus \delta \in T^{\prime \prime}$ implies $\delta(i, j) \in 2^{\alpha^{\prime}(i, j)} Z_{(2)}$. Conversely, $2^{\alpha^{\prime}(i, j)}\left(e_{5}(i, j) \oplus e_{2}(i, j)\right)$ satisfies the defining conditions of $T^{\prime \prime}$ whenever $i, j \in$ $\{4,5\}$.

\section{Submodules of $U$ and $V$}

We are thus lead to study in detail the submodule structure of $U$ and $V$. As an aside we note the following.

\subsection{Lemma. $U$ is generated by yxxy. $V$ is generated by $(t x)(y z)$.}

Proof. Firstly, use Corollary 4.13 to confirm that $U \varepsilon e$ is generated by $\mathbf{u}_{1}+\mathbf{u}_{2}$ $=\mathbf{w}_{1}+\mathbf{w}_{2}=\mathbf{a}_{1}+\mathbf{a}_{2}$. Next return to the definitions to see that $y x x y=\frac{1}{3}\left(-\mathbf{a}_{1}+\right.$ $\left.\mathbf{a}_{2}-\mathbf{a}_{3}\right)$, and use tables 4.3 to 4.5 to show that $(y x x y)\left[3\left(\xi_{1}-\xi_{3}\right) \varepsilon_{2} \sigma_{2} \varepsilon_{2}\right]=\mathbf{a}_{1}+$ $\mathbf{a}_{2}$ while $\left(\mathbf{a}_{1}+\mathbf{a}_{2}\right)\left[\frac{1}{3}\left(\varepsilon_{2} \sigma_{2} \varepsilon_{2}+\xi_{2}+2 \xi_{3}\right)\right]=y x x y$. This proves the first part; the second follows similarly.

5.2. Remark. This lemma is relevant in Section 2 of Fitzpatrick and Kovács (1982).

Corollary 4.13 makes it very easy to see just what $T$-submodules there are in Uee and $V \varepsilon e$.

As $T_{U}$ contains the diagonal elementary matrices $e_{5}(i, i)$, each $T$-submodule of $U \varepsilon e$ has a $Z_{(2)}$-basis consisting of scalar multiples of the basis elements $\mathbf{u}_{i}$ of $U \varepsilon e$. With the convention that $2^{\infty}=0$, we may therefore write each submodule in the form $\oplus 2^{u(i)} Z_{(2)} \mathbf{u}_{i}$ where each $u(i)$ is either $\infty$ or a nonnegative integer. Since $T_{U}$ has $Z_{(2)}$-basis $2^{\alpha^{\prime}(i, j)} e_{5}(i, j)$, such a $Z_{(2)}$-submodule admits $T$ if and only if

$$
u(i)+\alpha^{\prime}(i, j) \geqslant u(j) \text { for all } i, j \text { in }\{1, \ldots, 5\} \text {. }
$$

In particular, it follows that if $\oplus 2^{u(i)} Z_{(2)} \mathbf{u}_{i}$ is a $T$-submodule and one of the $u(i)$ is $\infty$ then all the $u(i)$ must be $\infty$, that is, the submodule is 0 . Also, $\mathbf{u}_{i} T=$ $\oplus 2^{\alpha^{\prime}(i, j)} Z_{(2)} \mathbf{u}_{j}$. In view of the connection we established by repeated use of $2.1,2.2$ between the $T$-submodules of $U \varepsilon e$ and the $Z_{(2)} G$-submodules of $U$, we therefore have the following. 
5.4. THEOREM. The nonzero $Z_{(2)} G$-submodules of $U$ are in one-to-one correspondence with the (ordered) 5-tuples

$$
(u(1), u(2), \ldots, u(5))
$$

of nonnegative integers satisfying 5.3; namely, the submodule of $U$ corresponding to such a 5-tuple is $\sum 2^{u(i)} \mathbf{u}_{i} Z_{(2)} G$. This submodule contains the submodule corresponding to $\left(u^{\prime}(1), \ldots, u^{\prime}(5)\right)$ if and only if $u(1) \leqslant u^{\prime}(1), \ldots, u(5) \leqslant u^{\prime}(5)$. More generally, the sum and the intersection of these two submodules correspond to the 5-tuples

$$
\left(\min \left\{u(1), u^{\prime}(1)\right\}, \ldots, \min \left\{u(5), u^{\prime}(5)\right\}\right)
$$

and

$$
\left(\max \left\{u(1), u^{\prime}(1)\right\}, \ldots, \max \left\{u(5), u^{\prime}(5)\right\}\right),
$$

respectively. In particular, $U$ corresponds to $(0, \ldots, 0)$, and $\mathbf{u}_{i} Z_{(2)} G$ to $\left(\alpha^{\prime}(i, 1), \ldots, \alpha^{\prime}(i, 5)\right)$.

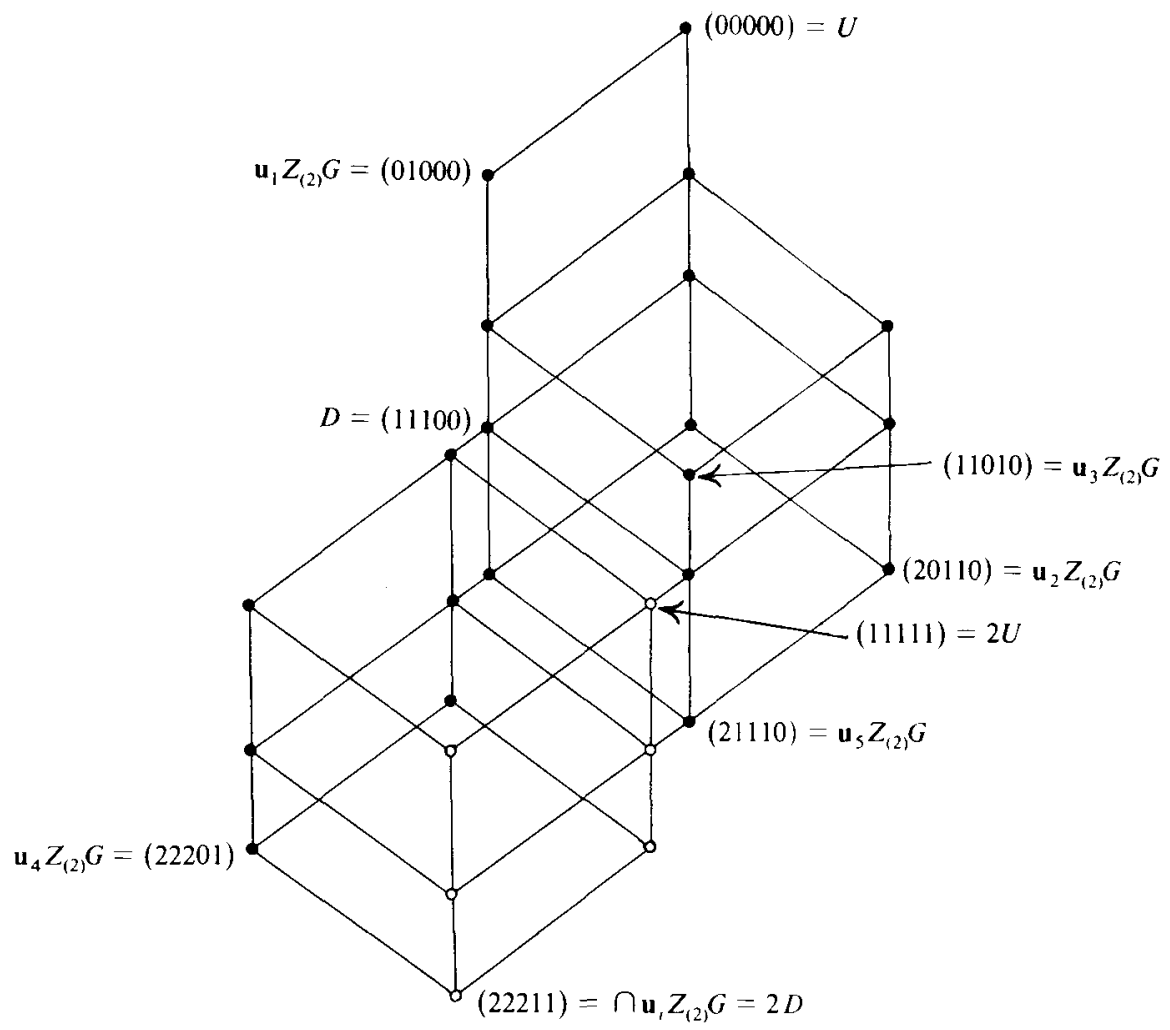

Submodule lattice of $U$ 
In order to visualize just which 5-tuples satisfy 5.3 and how the corresponding submodules relate to each other we have drawn the diagram of the sublattice of the submodule lattice $\delta(U)$ of $U$ consisting of the submodules which contain $\cap \mathbf{u}_{i} Z_{(2)} G$. For the moment, whenever convenient we identify a submodule with the corresponding 5-tuple; the omitted vertex labels of the diagram are readily obtainable by the rules for sums and intersections given in Theorem 5.4.

One helpful formal property of 5.3 is that if $(u(1), \ldots, u(5))$ satisfies it, so does $(u(1)+1, \ldots, u(5)+1)$ : this if the former corresponds to the module $M$, the 5-tuple corresponding to $2 M$ is the latter. Similarly, if $0 \neq N \in \delta(U)$ and $N=(u(1), \ldots, u(5))$, we may take $n=\min \{u(1), \ldots, u(5)\}$, define $M$ as $\sum 2^{u(i)-n} \mathbf{u}_{i} Z_{(2)} G$ so $N=2^{n} M$, and conclude that $M=(u(1)-n, \ldots, u(5)-n)$. As $u(j)=n$ for some $j$, for that $j$ we have $u(j)-n=0$ and hence $M \geqslant \mathbf{u}_{j} Z_{(2)} G$ $\geqslant \cap \mathbf{u}_{i} Z_{(2)} G$ : thus $M$ is one of the vertices of our diagram.

Heavy dots distinguish the vertices really needed in this context: those which correspond to submodules containing at least one $\mathbf{u}_{j} Z_{(2)} G$ (equivalently: those not contained in $2 U$ ). Note $N$ determines $n$ and $M$ uniquely.

Consider the translation of the plane of our diagram which takes $U$ to $2 U$. Observe that if the translate of a vertex $M$ is in the diagram, it is in fact $2 M$, and that each submodule contained in $2 U$ is such a translate. (In particular, $\cap \mathbf{u}_{i} Z_{(2)} G$ $=2 D$ with $D=(11100)$, a submodule of purely transient relevance.) Also, if $M$ and $M^{\prime}$ both have their translates in the diagram, $M$ and $M^{\prime}$ are joined by an edge if and only if $2 M$ and $2 M^{\prime}$ are.

We could build up the diagram of the whole lattice $\delta(U) \backslash\{0\}$ by applying the translation repeatedly, marking the images of all the vertices and edges, and labelling the $n$th translate of $M$ by $2^{n} M$. The discussion above proves that the vertices in this extended diagram would be in bijective correspondence with the elements of $\delta(U) \backslash\{0\}$, and it is clear that all edges drawn would be justified. The remaining point is that every necessary edge would be drawn this way. To see this, suppose $2^{n^{\prime}} M^{\prime}$ is a maximal submodule of $2^{n} M$ (where $M \$ 2 U$ and $M^{\prime} \$ 2 U$ ). If $n=n^{\prime}$, our instructions ensure that an edge is drawn joining $2^{n} M$ to $2^{n^{\prime}} M^{\prime}$. If $n>n^{\prime}$, then $M^{\prime}<2^{n-n^{\prime}} M \leqslant 2 U$, so this is excluded by our assumptions. Suppose then that $n<n^{\prime}$. Now $2^{n^{\prime}-n} M^{\prime}$ is a maximal submodule of $M$. We cannot have $2^{n^{\prime}-n} M^{\prime}+2 D=M$, for $M \$ 2 U$; on the other hand, $M \$ 2 U$ implies $2 D \leqslant M$ : so $2^{n^{\prime}-n} M^{\prime}$ must contain $2 D$. Thus $2^{n^{\prime}-n} M^{\prime}$ and $M$ are both vertices in the diagram we have actually drawn, so there is an edge joining them, and our instructions provide that one will be drawn joining $2^{n^{\prime}} M^{\prime}$ and $2^{n} M$. This establishes that the whole of $\delta(U) \backslash\{0\}$ can be visualized as indicated.

The submodules of $V$ are understood similarly, the rule of 5.3 being taken by

$$
v(i)+\alpha^{\prime}(i, j) \geqslant v(j) \text { for all } i, j \text { in }\{4,5\} \text {. }
$$


Of course, the nontrivial part of 5.5 may be written simply as

$$
v(4)-1 \leqslant v(5) \leqslant v(4)+1 .
$$

5.6. THEOREM. The nonzero $Z_{(2)} G$-submodules of $V$ are in one-to-one correspondence with the ordered pairs

$$
(v(4), v(5))
$$

of nonnegative integers satisfying 5.5; namely, the submodule of $V$ corresponding to such a pair is $2^{v(4)} \mathbf{v}_{4} Z_{(2)} G+2^{v(5)} \mathbf{v}_{5} Z_{(2)} G$. This submodule contains the submodule corresponding to $\left(v^{\prime}(4), v^{\prime}(5)\right)$ if and only if $v(4) \leqslant v^{\prime}(4)$ and $v(5) \leqslant v^{\prime}(5)$. More generally, the sum and the intersection of these two submodules correspond to

$$
\left(\min \left\{v(4), v^{\prime}(4)\right\}, \min \left\{v(5), v^{\prime}(5)\right\}\right)
$$

and

$$
\left(\max \left\{v(4), v^{\prime}(4)\right\}, \max \left\{v(5), v^{\prime}(5)\right\}\right),
$$

respectively. In particular, $V$ corresponds to $(0,0)$, and $\mathbf{v}_{i} Z_{(2)} G$ to $\left(\alpha^{\prime}(i, 4), \alpha^{\prime}(i, 5)\right)$.

The reason the theorem has been expressed in this elaborate form is that later we have to combine it with 5.4 , but of course it can be put much more concisely: the nonzero $Z_{(2)} G$-submodules of $V$ are just the $2^{i} V, 2^{j} \mathbf{v}_{4} Z_{(2)} G, 2^{k} v_{5} Z_{(2)} G$; two such submodules are comparable if and only if that is directly visible (using the relations $\left.2^{j} \mathbf{v}_{4} Z_{(2)} G \geqslant 2^{j+1} V \leqslant 2^{j} \mathbf{v}_{5} Z_{(2)} G\right)$ from the way we have written them.

The diagram $\delta(V) \backslash\{0\}$ is the following

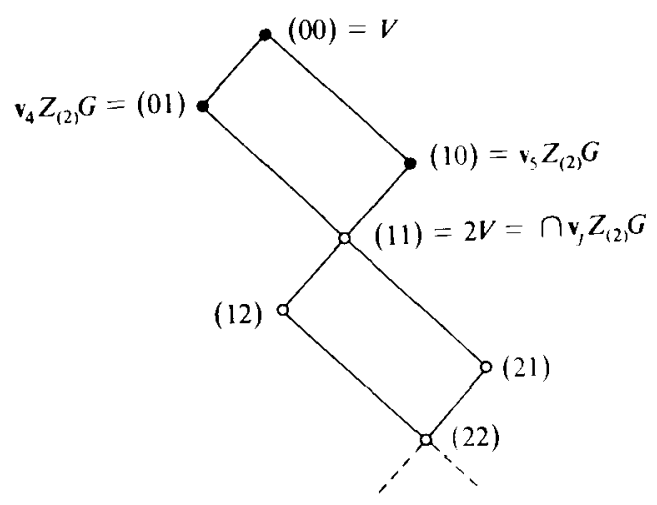




\section{Submodule structure of $W$}

Consider first the $T$-submodules of $U \varepsilon e \oplus V \varepsilon e$. Let $M$ be such a submodule. Put

$$
\begin{aligned}
& M \cap Z_{(2)} \mathbf{u}_{i}=2^{u(i)} Z_{(2)} \mathbf{u}_{i}, \\
& M \cap Z_{(2)} \mathbf{v}_{j}=2^{v(j)} Z_{(2)} \mathbf{v}_{j}
\end{aligned}
$$

(using the convention that $2^{\infty}=0$ when some of these intersections are 0 ) and

$$
N=\bigoplus_{i=1}^{5} 2^{u(i)} Z_{(2)} \mathbf{u}_{i} \oplus \bigoplus_{j=4}^{5} 2^{v(j)} Z_{(2)} \mathbf{v}_{j} .
$$

Corollary 4.12 shows that the $4 e_{5}(i, i)$ and the $4 e_{2}(j, j)$ are in $T_{U \oplus V}$, so we can conclude that

$$
4 M \leqslant N .
$$

Since $M \cap U \varepsilon e$ is a $T$-submodule and the $e_{5}(i, i)$ are all in $T_{U}$, we have $M \cap U \varepsilon e=\oplus\left(M \cap U_{\varepsilon e} \cap Z_{(2)} \mathbf{u}_{i}\right)=\oplus\left(M \cap Z_{(2)} \mathbf{u}_{i}\right)$, and a similar statement for $M \cap V \varepsilon e$. Therefore

$$
\begin{aligned}
& N=\left(M \cap U_{\varepsilon e}\right) \oplus\left(M \cap V_{\varepsilon}\right), \\
& \text { the } u(i) \text { satisfy } 5.3,
\end{aligned}
$$

and

the $v(j)$ satisfy 5.5 .

In particular if one of the $v(j)$ is $\infty$, so is the other, and then $M \cap V \varepsilon e=0$; thus $4 M \leqslant N=M \cap U \varepsilon e$ and hence $M \leqslant U \varepsilon e$. This case has been covered fully in the previous section, as has the case $M \leqslant V \varepsilon e$. For the submodules $M$ which require further investigation, we therefore have that all the $u(i)$ and $v(j)$ are nonnegative integers.

Next we exploit that $T_{U \oplus V}$ contains $e_{5}(i, i)$ when $i \leqslant 3$ and $e_{5}(j, j) \oplus e_{2}(j, j)$ when $j>3$, to conclude that

$$
M=\bigoplus_{i=1}^{3}\left(M \cap Z_{(2)} \mathbf{u}_{i}\right) \oplus \bigoplus_{j=4}^{5}\left(M \cap\left(Z_{(2)} \mathbf{u}_{j} \oplus Z_{(2)} \mathbf{v}_{j}\right)\right) .
$$

The first three summands are, of course, just the $\left.2^{u(i)} Z_{(2)}\right)_{i}$; the last two need to be looked at more closely.

Take the case $j=5$ first. We have

$$
\begin{aligned}
\left(M \cap\left(Z_{(2)} \mathbf{u}_{5} \oplus Z_{(2)} \mathbf{v}_{5}\right)\right) / & \left(2^{u(5)} Z_{(2)^{\mathbf{u}_{5}}} \oplus 2^{v(5)} Z_{(2)^{\mathbf{v}_{5}}}\right) \\
& \leqslant\left(Z_{(2)} \mathbf{u}_{5}\right) /\left(2^{u(5)} Z_{(2)^{2}} \mathbf{u}_{5}\right) \oplus\left(Z_{(2)} \mathbf{v}_{5}\right) /\left(2^{v(5)} Z_{(2)^{\mathbf{v}_{5}}}\right) .
\end{aligned}
$$


Here the right hand side is a direct sum of two cyclic 2-groups, and the left hand side is a subgroup which avoids both direct summands. Moreover, $2 e_{5}(5,5) \in$ $T_{U \oplus V}$ implies that this subgroup has exponent at most 2 . Forgetting our complex context for a moment, it is a trivial exercise that in such a direct sum there is only one such subgroup apart from 0. (Of course, if one or both cyclic direct summands degenerate, there is no nonzero subgroup of this kind.) We shall find it convenient to state the conclusion in the following form: $M \cap\left(Z_{(2)} \mathbf{u}_{5} \oplus Z_{(2)} \mathbf{v}_{5}\right)$ is generated (as additive group) by $2^{u(5)} Z_{(2)} \mathbf{u}_{5} \oplus 2^{v(5)} Z_{(2)} \mathbf{v}_{5}$ and $n\left(2^{u(5)-1} \mathbf{u}_{5}+\right.$ $2^{v(5)-1} v_{5}$ ) where $n$ is 0 or 1 , and if $n=1$ then $u(5) \geqslant 1$ and $v(5) \geqslant 1$.

Similar considerations apply to the case $j=4$. Again, we have to identify subgroups avoiding both direct summands in a direct sum of two cyclic 2-groups, but now we can only say that the subgroups of interest have exponent dividing 4 , so we find three nonzero possibilities (fewer when one or both summands have order less than 4). We consolidate the conclusions as follows.

6.1. Lemma. If $M$ is a T-submodule of $U \varepsilon e \oplus V \varepsilon e$, then as $Z_{(2)}$ module $M$ is generated by elements

$$
\begin{gathered}
2^{u(i)} \mathbf{u}_{i}, \quad 1 \leqslant i \leqslant 5, \\
2^{v(j)} \mathbf{v}_{j}, \quad 4 \leqslant j \leqslant 5, \\
k\left(2^{u(4)-1} \mathbf{u}_{4}+2^{v(4)-1} \mathbf{v}_{4}\right), \\
l\left(2^{u(4)-2} \mathbf{u}_{4}+2^{v(4)-2} \mathbf{v}_{4}\right), \\
m\left(2^{u(4)-2} \mathbf{u}_{4}-2^{v(4)-2} \mathbf{v}_{4}\right), \\
n\left(2^{u(5)-1} \mathbf{u}_{5}+2^{v(5)-1} \mathbf{v}_{5}\right),
\end{gathered}
$$

where

(1) the $u(i)$ and $v(j)$ are nonnegative integers or $\infty$, subject to 5.3 and 5.5, and if any $\infty$ occur then $k=l=m=n=0$;

(2) $k, l, m, n \in\{0,1\}$ and $k+l+m \leqslant 1$;

(3) if $k=1$ then $u(4) \geqslant 1$ and $v(4) \geqslant 1$, if $l+m=1$ then $u(4) \geqslant 2$ and $v(4) \geqslant 2$, if $n=1$ then $u(5) \geqslant 1$ and $v(5) \geqslant 1$.

We now consider any $Z_{(2)}$-submodule $M$ of $U \varepsilon e \oplus V \varepsilon e$ generated by the elements listed in 6.1 with the parameters satisfying the conditions of 6.1. For any elements of $U \varepsilon e \oplus V \varepsilon e$ expressed in terms of the basis $\mathbf{u}_{1}, \ldots, \mathbf{v}_{5}$, it is straightforward to decide whether it lies in $M$. Thus we are well prepared to find a necessary and sufficient set of conditions (in terms of the parameters $u(1), \ldots, n$ of $M$ ) for $M$ to admit $T_{U \oplus V}$. Indeed, the conditions already imposed on the parameters 
clearly ensure that $M$ admits the following subset of $T_{U \oplus V}$ :

$$
\begin{aligned}
\left\{2^{\alpha^{\prime}(i, j)} e_{5}(i, j) \mid 1 \leqslant i \leqslant 3\right\} \cup & \left\{e_{5}(j, j) \oplus e_{2}(j, j) \mid 4 \leqslant j \leqslant 5\right\} \\
& \cup\left\{4 e_{5}(4,4), 4 e_{5}(4,5), 4 e_{5}(5,4), 2 e_{5}(5,5)\right\} .
\end{aligned}
$$

Corollary 4.12 shows that the union of this set with

$$
\left\{2^{\alpha^{\prime}(i, j)} e_{5}(i, j) \mid i>3 \geqslant j\right\} \cup\left\{2 e_{5}(4,5) \oplus 2 e_{2}(4,5), 2 e_{5}(5,4) \oplus 2 e_{2}(5,4)\right\}
$$

generates $T_{U \oplus V}$. Thus we need only write down the conditions which express that $M$ contains the image of each of its given generators by each of the (eight) elements of the last displayed subset. The list of simple conditions so obtained is long and highly redundant; we shall not write it out here. Instead, we include a shorter but trivially equivalent list in the following statement, which also takes advantage of the connection established, via 2.1 and 2.2 , between $T$-submodules of $U \varepsilon e \oplus V \varepsilon e$ and $Z_{(2)} G$-submodules of $U \oplus V$.

6.2. THEOREM. The $Z_{(2)} G$-submodules of $U \oplus V$ are in one-to-one correspondence with the ordered 11-tuples

$$
(u(1), \ldots, u(5), v(4), v(5) ; k, l, m, n)
$$

which satisfy conditions (1), (2), (3) of 6.1 and also the following:

(4) if $k=1$ then $u(4)+1 \geqslant \max \{u(1), u(2), u(3)\}$ and either $u(4) \geqslant u(5)$ and $v(4) \geqslant v(5)$ or $u(4)=u(5)-1$ and $v(4)=v(5)-1$ and $n=1$;

(5) if $l+m=1$ then $u(4) \geqslant \max \{u(1), u(2), u(3)\}$ and either $u(4)=u(5)+1$ and $v(4)=v(5)+1$ or $u(4)=u(5)$ and $v(4)=v(5)$ and $n=1$;

(6) if $n=1$ then $u(5) \geqslant \max \{u(1)-1, u(2), u(3)\}$ and either $u(4) \leqslant u(5)$ and $v(4) \leqslant v(5)$ or $u(4)=u(5)+1$ and $v(4)=v(5)+1$ and $k+l+m=1$.

The submodule corresponding to these parameters is generated, as $Z_{(2)} G$-module, by the elements listed in 6.1. It contains the submodule corresponding to $\left(u^{\prime}(1), \ldots, n^{\prime}\right)$ if and only if the following conditions hold:

(7) $u(i) \leqslant u^{\prime}(i)$ for $1 \leqslant i \leqslant 5, v(j) \leqslant v^{\prime}(j)$ for $4 \leqslant j \leqslant 5$;

(8) if $k^{\prime}=1$ then either $u(4) \leqslant u^{\prime}(4)-1$ and $v(4) \leqslant v^{\prime}(4)-1$ or $u(4)=u^{\prime}(4)$ and $v(4)=v^{\prime}(4)$ and $k+l+m=1$;

(9) if $l^{\prime}+m^{\prime}=1$ then either $u(4) \leqslant u^{\prime}(4)-2$ and $v(4) \leqslant v^{\prime}(4)-2$ or $u(4)=$ $u^{\prime}(4)-1$ and $v(4)=v^{\prime}(4)-1$ and $k+l+m=1$ or $u(4)=u^{\prime}(4)$ and $v(4)=v^{\prime}(4)$ and $l=l^{\prime}, m=m^{\prime}$;

(10) if $n^{\prime}=1$ then either $u(5) \leqslant u^{\prime}(5)-1$ and $v(5) \leqslant v^{\prime}(5)-1$ or $u(5)=u^{\prime}(5)$ and $v(5)=v^{\prime}(5)$ and $n=1$. 
The conditions (7)-(10) are also obtained in the context of 6.1 and $U_{\varepsilon e} \oplus V_{\varepsilon e}$. Their derivation is merely tedious and is omitted. The submodule lattice $\delta(U \oplus V)$ is clearly much more complicated than $\delta(U)$ or $\delta(V)$ : it contains the direct product of these two lattices (as the sublattice consisting of the submodules with $k=l=m=n=0$ ) but it is not distributive and this makes it hard to visualize; we present no diagrams. The parameters for the sum and intersection of two submodules may be calculated from the parameters of the components, but the best algorithms we could find for these calculations are rather complicated so we do not include them.

The final step in this section is to use 4.10 to conclude that $4 W \varepsilon e$ is the submodule of $U \varepsilon e \oplus V \varepsilon e$ generated by $\mathbf{u}_{4}+3 \mathbf{v}_{4}$. Theorem 6.2 may now be used to identify $4 W$ as the submodule of $U \oplus V$ corresponding to $(2,2,2,2,2,2,2$; $0,0,1,1)$. We can use 6.2 again to find the submodules of $4 W$. As $W \rightarrow 4 W$, $w \mapsto 4 w$ is an isomorphism that information can be translated into the following description of all submodules of $W$.

6.3. THEOREM. The $Z_{(2)} G$-submodules of $W$ are in one-to-one correspondence with the ordered 11-tuples

$$
(u(1), \ldots, u(5), v(4), v(5) ; k, l, m, n)
$$

which satisfy the conditions (1), (2) of 6.1, the conditions (4), (5), (6) of 6.2 and the following conditions:

(11) if $k=1$ then either $u(4) \geqslant 1$ and $v(4) \geqslant 1$ or $u(4)=v(4)$;

(12) if $l=1$ then either $u(4) \geqslant 2$ and $v(4) \geqslant 2$ or $u(4)=v(4)=1$;

(13) if $m=1$ then either $u(4) \geqslant 2$ and $v(4) \geqslant 2$ or $u(4)=v(4)$;

(14) if $n=1$ then either $u(5) \geqslant 1$ and $v(5) \geqslant 1$ or $u(5)=v(5)$.

The submodule corresponding to these parameters is generated, as $Z_{(2)} G$-module, by the elements listed in 6.1. It contains the submodule corresponding to the parameters $\left(u^{\prime}(1), \ldots, n^{\prime}\right)$ if and only if conditions (7), (8), (9), (10) of 6.2 are satisfied.

Note that the conditions (11), (12), (13), (14) which replaced (3) of 6.1, allow four problematic expressions to occur in the list of generators of submodules: namely $2^{-1} \mathbf{u}_{4} \pm 2^{-1} \mathbf{v}_{4}, 2^{-2} \mathbf{u}_{4}-2^{-2} \mathbf{v}_{4}$, and $2^{-1} \mathbf{u}_{5}+2^{-1} \mathbf{v}_{5}$. These should be handled with care, as for instance $2^{-2} \mathbf{u}_{4}$ is not an element of $W$. They can be interpreted however, by translating back to the basis $\left\{\mathbf{w}_{1}, \ldots, \mathbf{w}_{7}\right\}$ of $W_{\varepsilon e}$ : this minor inconvenience seems preferable to making the formalism of 6.3 still more complicated. 
As we have seen $U$ and $V$ are the only isolated proper nonzero $Z_{(2)} G$-submodules of $W$. Their parameters are:

$$
\begin{aligned}
& U=(0,0,0,0,0, \infty, \infty ; 0,0,0,0), \\
& V=(\infty, \infty, \infty, \infty, \infty, 0,0 ; 0,0,0,0)
\end{aligned}
$$

while

$$
W=(0,0, \ldots, 0 ; 0,0,1,1) .
$$

Of course,

$$
0=(\infty, \infty, \ldots, \infty ; 0,0,0,0) .
$$

It is straightforward to recognise from the parameters, just by the occurrences of $\infty$, what the isolator of any particular $Z_{(2)} G$-module is. Also the exponent of the quotient of the isolator modulo the submodule may readily be determined: for example, if the submodule has parameters $(u(1), \ldots, n)$ with no $\infty$ among them, then the exponent of the quotient of $W$ over this submodule is the maximum of the following list of numbers:

$2^{u(1)}, 2^{u(2)}, 2^{u(3)}$;

$2^{u(4)+2}, 2^{v(4)+2}$ unless $u(4)=v(4)$ and $k+l+m=1$ in which case these two numbers are replaced by $2^{u(4)+k+l}$;

$2^{u(5)+1}, 2^{v(5)+1}$ unless $u(5)=v(5)$ and $n=1$ in which case these two numbers are replaced by $2^{u(5)}$.

These examples illustrate the kind of information one can derive from 6.3. It is also true that the long argument which culminated in 6.3 implicitly enables one to decide, for any element of $W$ and for any submodule given by its parameters, whether the element lies in the submodule. We shall not undertake the task of making this claim formal and elaborating a general algorithm.

\section{The last term of $F$}

At last we are ready to turn to the project outlined in the introduction. Let $F$ be the free nilpotent group of class 4 freely generated by $x, y, z, t$ : our task is to determine the $2^{\prime}$-isolated fully invariant subgroups of $F$ which lie in the last nontrivial term $N_{3}=\mathfrak{N}_{3}(F)$ of the lower central series of $F$. As we mentioned in the introduction, the Magnus-Witt argument given in Section 3 of Kovács (1978) admits an obvious adaptation which yields that the 2 -isolated fully invariant subgroups of $F$ contained in $N_{3}$ are in one-to-one correspondence with the $Z_{(2)} G$-submodules of the module $W$ we have been studying so far. Moreover, the nature of that correspondence is that if Lie ring sums are replaced by group products and Lie products by group commutators, most of the detail we have 
uncovered can be translated from $W$ to $N_{3}$. We have deliberately used $x, y, z, t$ in both contexts. Expressing the $\mathbf{u}_{i}, \mathbf{v}_{j}$ of $W$ in terms of $x, y, z, t$ allows one to identify the corresponding elements of $N_{3}$, to which we transfer the names $\mathbf{u}_{1}, \ldots, \mathbf{v}_{5}$ : from now on,

$$
\begin{aligned}
\mathbf{u}_{1}= & {[y, x, x, y][y, x, x, x][x, y, y, y]^{-1}, } \\
\mathbf{u}_{2}= & {[y, x, x, y][y, x, x, x]^{-1}[x, y, y, y]^{-2}, } \\
\mathbf{u}_{3}= & {[y, x, x, z]^{-3}[z, x, x, y][y, x, x, z]^{3}[z, y, y, x]^{-3}[x, y, y, z] } \\
& \cdot[z, y, y, x]^{3}[x, z, z, y]^{-3}[y, z, z, x][x, z, z, y]^{3}, \\
\mathbf{u}_{4}= & {[y, x, x, z]^{4}[y, x, x, z]^{-3}, } \\
\mathbf{u}_{5}= & {[t, x, y, z]^{2}[t, y, z, x]^{2}[t, z, x, y]^{2}[t, x, y, z]^{-1}[t, y, z, x]^{-1} } \\
& \cdot[t, z, x, y]^{-1}, \\
\mathbf{v}_{4}= & {[y, x, x, z], } \\
\mathbf{v}_{5}= & {[t, x, y, z][t, y, z, x][t, z, x, y] . }
\end{aligned}
$$

(Commutators without double commas are to be read as left-normed, so $[y, x, x, y]=[[[y, x], x], y]$, while $[y, x, x, z]$ stands for $[[y, x],[x, z]]$, and so on.) The translation of 6.3 is the following.

7.1. THEOREM. The 2'-isolated fully invariant subgroups of $F$ contained in $\mathfrak{R}_{3}(F)$ are in one-to-one correspondence with the ordered 11-tuples

$$
(u(1), \ldots, u(5), v(4), v(5) ; k, l, m, n)
$$

which satisfy conditions (1), (2) of 6.1, (4), (5), (6) of 6.2, and (11), (12), (13), (14) of 6.3. The subgroup corresponding to these parameters is generated (as fully invariant subgroup of $F$ ) by the following elements:

$$
\begin{aligned}
& \mathbf{u}_{i}^{2^{u(i)}} \text { with } 1 \leqslant i \leqslant 5 \text {, } \\
& \mathbf{v}_{j}^{2^{v(j)}} \text { with } 4 \leqslant j \leqslant 5 \text {, } \\
& \left(\mathbf{u}_{4}^{2^{u(4)-1}} \mathbf{v}_{4}^{2^{v(4)-1}}\right)^{k} \text {, } \\
& \left(\mathbf{u}_{4}^{2^{u(4)-2}} \mathbf{v}_{4}^{2^{2(4)-2}}\right)^{\prime}, \\
& \left(\mathbf{u}_{4}^{2^{u(4)-2}} \mathbf{v}_{4}^{-2^{v(4)-2}}\right)^{m} \text {, } \\
& \left(\mathbf{u}_{5}^{2^{u(5)-1}} \mathbf{v}_{5}^{2^{u(5)-1}}\right)^{n} \text {, } \\
& {[y, x, x, y]^{2 u} \text { where } u=\max \{u(1), \ldots, u(5)\} \text {, }} \\
& {[t, x, y, z]^{2^{v}} \text { where } v=\max \{v(4), v(5)\} .}
\end{aligned}
$$

It contains the fully invariant subgroup corresponding to the parameters $\left(u^{\prime}(1), \ldots, n^{\prime}\right)$ if and only if conditions (7), (8), (9), (10) of 6.2 are satisfied. 
The convention $2^{\infty}=0$ remains in force. The explanatory paragraph after 6.3 translates as follows:

$$
\begin{aligned}
\mathbf{u}_{4}^{2^{-1}} \mathbf{v}_{4}^{2^{-1}} & =[y, x, x, z]^{2}[y, x, x, z]^{-1}, \\
\mathbf{u}_{4}^{2^{-1}} \mathbf{v}_{4}^{-2^{-1}} & =[y, x, x, z]^{2}[y, x, x, z]^{-2}, \\
\mathbf{u}_{4}^{2^{-2}} \mathbf{v}_{4}^{-2^{-2}} & =[y, x, x, z][y, x, x, z]^{-1}, \\
\mathbf{u}_{5}^{2^{-1}} \mathbf{v}_{5}^{2^{-1}} & =[t, x, y, z][t, y, z, x][t, z, x, y]
\end{aligned}
$$

give the interpretation of formally nonsensical expressions which sometimes appear in the list of generators in 7.1.

Let $M$ be the fully invariant subgroup closure of $[y, x, x, y]$ and $F^{\prime \prime}$ the second derived group which is the fully invariant subgroup closure of $[t, x, y, z]$. By Fitzpatrick and Kovács (1982), $M$ is isolated (where our Lemma 5.1 was used in this context), so $M$ is the verbal subgroup corresponding to the variety $\Re_{3}^{(2)} \cap \Re_{4}$. Moreover in the same paper it was shown that $N_{3}^{4} \leqslant M F^{\prime \prime}$ (where if $A$ is any abelian subgroup of $F$ we write $A^{k}$ for $\left\{a^{k} \mid a \in A\right\}$ ).

Let $H$ be the $2^{\prime}$-isolated fully invariant subgroup of $F$ corresponding to the submodule of $W$ with parameters $(u(1), \ldots, n)$. Clearly, $H$ is the $2^{\prime}$-isolated fully invariant subgroup closure of the set of elements listed in 7.1 with the omission of the last two; that is, $H$ is the subgroup of $F$ generated by this set of elements under the operations of applying endomorphisms of $F$ and taking $n$th roots where possible for each odd (positive) integer $n$. Let $H_{0}$ be the isolator of $H$ (see the introduction) and $H_{1}$ the fully invariant subgroup closure of the complete set of elements listed in 7.1. Then $H_{1} \leqslant H \leqslant H_{0}$ and $H_{0}$ is also the isolator of $H_{1}$. Moreover there is a nonnegative integer $r$ such that $H_{0}^{2^{r}} \leqslant H_{1}$ : by the previous paragraph, if $H_{0}=M$ then $r=u$, if $H_{0}=F^{\prime \prime}$ then $r=v$ and if $H_{0}=N_{3}$ then $r=2+\max \{u, v\}$.

Now if $w$ belongs to $H$ then $w$ can be expressed as $w=w_{1}^{1 / n}$ where $w_{1}$ is a product of (integer) powers of images of the generators of $H$ (as $2^{\prime}$-isolated fully invariant subgroup) and $n$ is an odd positive integer. Hence $w_{1}=w^{n}$ is an element of $H_{1}$. But $w^{2^{r}} \in H_{1}$ and so $w \in H_{1}$ also. Thus $H=H_{1}$ and the derivation of 7.1 is complete.

The parameters of $N_{3}$ are $(0,0, \ldots, 0 ; 0,0,1,1)$, those of $M$ are $(0, \ldots, 0, \infty, \infty$; $0, \ldots, 0)$ and those of $F^{\prime \prime}$ are $(\infty, \ldots, \infty, 0,0 ; 0, \ldots 0)$. Clearly, the factor group $H_{0} / H$ has exponent dividing

$$
\begin{array}{ll}
2^{u} & \text { when } u<\infty=v, \\
2^{v} & \text { when } u=\infty>v, \\
2^{2+\max \{u, v\}} & \text { when } u<\infty>v .
\end{array}
$$

(Of course this factor is trivial when $u=v=\infty$.) Thus the requirements of the programme laid out in Fitzpatrick and Kovács (1982) are fulfilled. 


\section{References}

H. Boerner (1963), Representations of groups (North-Holland, Amsterdam).

Patrick Fitzpatrick (1980), Varieties of nilpotent groups of class four (Ph.D. thesis, Australian National University, Canberra).

Patrick Fitzpatrick and L. G. Kovács (1983), 'Varieties of nilpotent groups of class four (I)', $J$. Austral. Math. Soc. Ser. A 35, 59-73.

L. G. Kovác, (1978), 'Varieties of nilpotent groups of small class', Topics in algebra (Proc. 18th SRI, Lecture Notes in Mathematics, 697, pp. 205-229. Springer-Verlag, Berlin, Heidelberg, New York).

Hanna Neumann (1967), Varieties of groups (Ergebnisse der Mathematik und ihrer Grenzgebiete, 37. Springer-Verlag, Berlin, Heidelberg, New York).

G. E. Wall (1978), 'Lie methods in group theory', Topics in algebra (Proc. 18th SRI, Lecture Notes in Mathematics, 697, pp. 137-713. Springer-Verlag, Berlin, Heidelberg, New York).

\section{Department of Mathematics}

University College

Cork

Ireland 